\title{
1. Zum Stand der Forschung
}

\subsection{Was sind Bürgerwehren?}

\subsubsection{Historische Bürgerwehren: Zum Wandel des Begriffs "Bürgerwehr"}

Der Begriff »Bürgerwehr« geht im Deutschen zurück auf die im 18. und 19. Jahrhundert gegründeten militärischen Stadtgarden. Damals galt eine Waffenpflicht der Bürger zur Verteidigung ihrer Stadt, und daraus entstanden vielerorts Verteidigungseinrichtungen, die sich Bürgerwehr nannten. ${ }^{1}$ Mit der Entwicklung stehender Heere wurden die Bürgerwehren jedoch überflüssig. Heute existieren diese historischen Bürgerwehren nur noch als folkloristische Einrichtungen, ohne wirkliche sicherheitspolitische Bedeutung. ${ }^{2}$

In Wörterbüchern findet sich bis heute die ursprüngliche Definition als "Gesamtheit der von Bürgern einer Gemeinde gebildeten bewaffneten Einheiten . $^{3}$ Die Bedeutung des Begriffs »Bürgerwehr« hat sich jedoch im Vergleich

Vgl. Bergien, Rüdiger (Hg.) (2010): Spießer, Patrioten, Revolutionäre. Militärische Mobilisierung und gesellschaftliche Ordnung in der Neuzeit. Göttingen: Vandenhoeck \& Ruprecht. Und: Pröve, Ralf (2000): Stadtgemeindlicher Republikanismus und die »Macht des Volkes«. Zivile Ordnungsformationen und kommunale Leitbilder politischer Partizipation in den deutschen Staaten vom Ende des 18. bis zur Mitte des 19. Jahrhunderts. Göttingen: Vandenhoeck \& Ruprecht.

2 Vgl. Kranz, Rolf (1996): Gott-Heimat-Vaterland. Über das Selbstverständnis heutiger badischer Bürgerwehren. Friesenheim: Kranz.

3 Vgl. duden.de, Stichwort Bürgerwehr: https://www.duden.de/rechtschreibung/Buerger wehr (Zugriff am 2.1.2021). Meyers Lexikon verweist unter Bürgerwehr auf das Stichwort Volksbewaffnung: http://woerterbuchnetz.de/cgi-bin/WBNetz/wbgui_py?sigle=M eyers\&mode=Vernetzung\&lemid=IB10519\#XIB10519 (Zugriff am 2.1.2021). Ebenso bei Wikipedia: de.wikipedia.org/wiki/Bürgerwehr (Zugriff am 2.1.2021). 
dieser ursprünglichen Bedeutung zu dem, was heute in der gesellschaftlichen Debatte unter Bürgerwehren verstanden wird, stark verändert. ${ }^{4}$ In der heutigen Bedeutung ist lediglich der Selbstschutz durch Privatpersonen erhalten geblieben.

Im Gegensatz zu den zeitgenössischen Bürgerwehren sind die historischen Bürgerwehren aus dem 19. Jahrhundert wissenschaftlich gut erfasst. ${ }^{5}$ Insbesondere zur Rolle der Bürgerwehren in der Märzrevolution finden sich zahlreiche Arbeiten. ${ }^{6}$ Mit dem Übergang ins 20. Jahrhundert verändert sich die Bedeutung des Begriffs "Bürgerwehren«. Nach dem Ersten Weltkrieg gab es nichtstaatliche Gewaltgruppen, die sich als Bürgerwehren bezeichneten. So waren es Mitglieder der Wilmersdorfer Bürgerwehr, die Rosa Luxemburg und Karl Liebknecht festnahmen und sie den Freikorps übergaben, die sie ermordeten. ${ }^{7}$

Diese faschistischen Gruppen, die sich im frühen 20. Jahrhundert als Bürgerwehren bezeichnen, sind Symptom einer polarisierten politischen Lage, in der der Straßenraum zum Schauplatz politischer Kämpfe wurde. Kommunistische und faschistische Bewegungen konkurrierten um die Vorherrschaft im öffentlichen Raum. Dieser sollte nicht nur visuell mit Plakaten und Flaggen beherrscht werden, sondern auch durch (uniformierte) physische Präsenz. Ab

4 Vgl. hierzu Hoffmann, Anika (2019): Bürgerwehren und ihre Bedeutung im öffentlichen Raum. Abweichendes Verhalten als kriminologisch relevantes Phänomen. Wiesbaden: VS Verlag. S. $16 \mathrm{f}$.

5 Vgl. Bergien, Rüdiger (Hg.) (2010): Spießer, Patrioten, Revolutionäre. Militärische Mobilisierung und gesellschaftliche Ordnung in der Neuzeit. Göttingen: Vandenhoeck \& Ruprecht. Und: Pröve, Ralf (2000): Stadtgemeindlicher Republikanismus und die »Macht des Volkes«. Civile Ordnungsformationen und kommunale Leitbilder politischer Partizipation in den deutschen Staaten vom Ende des 18. bis zur Mitte des 19. Jahrhunderts. Göttingen: Vandenhoeck \& Ruprecht. Zu den Defiziten der wissenschaftlichen Erfassung von Militärgeschichte seit 1945 vgl. Pröve, Ralf (2011): Bürgergewalt und Staatsgewalt. Bewaffnete Bürger und vorkonstitutionelle Herrschaft im frühen 19. Jahrhundert. In: Lüdtke/Reinke/Sturm (Hg.) (2011): Polizei, Gewalt und Staat im 20. Jahrhundert. Wiesbaden: VS Verlag. S. $63 \mathrm{f}$.

6 Vgl. insbes. Güthler, Franziska (2003): Heilbronn 1848/49. Die Rolle von Militär und Bürgerwehr in der Revolution. Heilbronn: Stadtarchiv Heilbronn. Und: Beiring, Ingrid (2009): »Zum Schutze des Eigenthums und der Person«: Bürgerwehren in der Revolution von 1848/49 im Westmünsterland. Vreden: Landeskundliches Institut Westmünsterland.

7 Vgl. Quent, Matthias (2016): Bürgerwehren. Hilfssheriffs oder inszenierte Provokation? Studie für die Amadeus Antonio Stiftung. Cottbus: Druckzone. S. 5. 
1933 wurde der Straßenraum von der NSDAP strategisch zur Repräsentation und Visualisierung von Macht verwendet. ${ }^{8}$

\subsubsection{Zeitgenössische Bürgerwehren in Deutschland - Eine Definition}

In den 1990er Jahren gründeten sich an zahlreichen Orten Bürgerinitiativen, die sich für Sicherheit engagierten. Insbesondere Ronald Hitzler analysierte dieses Phänomen ${ }^{9}$ und beschreibt diese Bürgerwehren als »neue soziale Sicherheits-Bewegung «. ${ }^{10}$ Den mentalen Nährboden für diese Gruppierungen stellt nach Hitzler ein in den 1990ern verstärkt von der Politik geäußerter Appell an die Bürger*innen dar, Mitverantwortung für die öffentliche Sicherheit zu übernehmen und »damit eine >Kultur des Hinsehens « wiederzubeleben wider die in modernen Gesellschaften angeblich grassierende >Unkultur des Wegschauens « ${ }^{11}$

Parallel zu diesen selbstorganisierten Gruppen fand in den 1990er Jahren in einigen Bundesländern eine Einbindung von Bürger*innen in die Polizei-

Vgl. Schmidt, Daniel (2011): Die Straße beherrschen, die Stadt beherrschen. Sozialraumstrategien und politische Cewalt im Ruhrgebiet 1929-1933. In: Lüdtke/Reinke/Sturm (Hg.) (2011): Polizei, Gewalt und Staat im 20. Jahrhundert. Wiesbaden: VS Verlag. S. $227 f$.

9 Vgl. Hitzler, Ronald (1993): Bürger machen mobil. Über die neue soziale SicherheitsBewegung. In: Forschungsjournal Neue Soziale Bewegungen 3-4. S. 16-27. Sowie Hitzler, Ronald (1994): Die neuen Vigilanten. Über Formen der Bewältigung alltäglicher Verunsicherung In: Bürgerrechte \& Polizei 48: 2. S. 67-71. Und: Hitzler, Ronald (1996): Der in die Polizeiarbeit eingebundene Bürger. Zur symbolischen Politik mit der bayrischen Sicherheitswacht. In: Reichertz, Jo/Schröer, Norbert (Hg.): Qualitäten polizeilichen Handelns. Beiträge zu einer verstehenden Polizeiforschung. Opladen: Westdeutscher Verlag. S. 30-47. Siehe außerdem: Ooyen, Robert Christian van (2007): Öffentliche Sicherheit und Freiheit. Studien zu Staat, Polizei und wehrhafter Demokratie. Baden-Baden: Nomos. S. 123. Sowie: Hohmeyer, Christine (2000): Wachsame Nachbarn. Lokale Gemeinschaften im Dienst der Sicherheit. In: Bürgerrechte \& Polizei/CILIP 66 (2/2000), S. 51-60. Außerdem befasst sich Thomas Schmidt-Lux mit Ausläufern dieses Phänomens in den 2000ern: Vgl. Schmidt-Lux, Thomas (2012): Vigilantismus. Ein Phänomen der Grenze? In: Kriminologisches Journal, Heft 2, 2012. S. 118-132.

10 Vgl. Hitzler, Ronald (1993): Bürger machen mobil. Über die neue soziale SicherheitsBewegung. In: Forschungsjournal Neue Soziale Bewegungen 3-4. S. 16-27.

11 Hitzler, Ronald (1996): Der in die Polizeiarbeit eingebundene Bürger. Zur symbolischen Politik mit der bayrischen Sicherheitswacht. In: Reichertz, Jo/Schröer, Norbert (Hg.): Qualitäten polizeilichen Handelns. Beiträge zu einer verstehenden Polizeiforschung. Opladen: Westdeutscher Verlag. S. 34. 
arbeit statt. Unter den Bezeichnungen "Sicherheitspartnerschaften« (Brandenburg), »Sicherheitswacht « (Bayern und Sachsen) und »Freiwilliger Polizeidienst « (Baden-Württemberg und Hessen), ${ }^{12}$ werden Bürger*innen bis heute von den lokalen Behörden ausgesucht und mit hilfspolizeilichen Aufgaben betraut. Sie werden geschult und bekommen teilweise eine Aufwandsentschädigung für ihre Tätigkeiten.

Auf einer rechtlichen Ebene fußt das Engagement der Bürger"innen auf dem sogenannten Jedermannsrecht, in $\$ 127$ der Strafprozessordnung als »Vorläufige Festnahme« festgehalten«, nach dem jede"r Bürger"in, wenn er oder sie ein Verbrechen beobachtet, das Recht hat, einen Verdächtigen so lange festzuhalten, bis die Polizei kommt.

»Wird jemand auf frischer Tat betroffen oder verfolgt, so ist, wenn er der Flucht verdächtig ist oder seine Identität nicht sofort festgestellt werden kann, jedermann befugt, ihn auch ohne richterliche Anordnung vorläufig festzunehmen. $\ll^{13}$

Während die Befugnisse der unabhängig vom Staat organisierten Gruppen sowie die der in Sicherheitspartnerschaften eingebundenen Bürger"innen nicht über das Jedermannsrecht hinausgehen, sieht das Konzept der Bayerischen Sicherheitswacht darüber hinaus weitere Handlungsmöglichkeiten vor. Hier haben die Bürger*innen das Recht, Leute anzuhalten, Personalien festzustellen und Platzverweise zu erteilen. ${ }^{14}$

In den letzten Jahren, insbesondere seit 2016, ist eine neue Gründungswelle von Bürgerwehren zu verzeichnen. Diese Gruppen bezeichnen sich selber bei weitem nicht alle als Bürgerwehren, auch die Bezeichnungen »Bürgerstreife« oder »Bürgerinitiative« sind verbreitet. Sie sind in der Regel nicht in Sicherheitspartnerschaften eingebunden und unterstehen nicht der Polizei. Die Anzahl dieser neuen Bürgerwehren ist schwer zu schätzen. Es existieren keine offiziellen Erhebungen staatlicher Behörden. Thomas Schmidt-Lux

12 Vgl. http://www.sicherheit-braucht-partner.brandenburg.de/cms/detail.php/bb1.c.5071 23.de (Zugriff am 2.1.2021). https://www.polizei.sachsen.de/de/3620.htm (Zugriff am 2.1.2021). https://www.polizei.bayern.de/wir/sicherheitswacht/ (Zugriff am 2.1.2021).

13 Vgl. § 127 Strafprozessordnung Absatz 1. Online unter: https://dejure.org/gesetze/StPO /127.html (Zugriff am 2.1.2021).

14 Vgl. Ooyen, Robert Christian van (2007): Öffentliche Sicherheit und Freiheit. Studien zu Staat, Polizei und wehrhafter Demokratie. Baden-Baden: Nomos. S. 123. 
geht davon aus, dass es im Frühjahr 2016 zwischen 150 und 200 Bürgerwehren oder bürgerwehrähnliche Gruppen gab. ${ }^{15}$ Anika Hoffmann erfasst in ihrer statistischen Erhebung Facebook-Gruppen von Bürgerwehren und verzeichnet am 01.12.2016 456 Facebook-Gruppen mit 17.482 Mitgliedern, die unter dem Namen »Bürgerwehr « online abrufbar sind. ${ }^{16}$ Diese Erhebung bezieht sich jedoch nur auf diejenigen Gruppen, die das Wort »Bürgerwehr« für sich verwenden und in Facebook-Gruppen vernetzt sind. Einige dieser Gruppen existieren als reine Online-Gruppen und treffen sich nicht im öffentlichen Raum, andere agieren im öffentlichen Raum, ohne sich auf Facebook in einer Gruppe mit dem Begriff »Bürgerwehr« im Namen zu vernetzen. Sie verabreden sich beispielsweise über Messengerdienste. Dies trifft auf zwei der in dieser Studie untersuchten Bürgerwehren zu.

Die große Bandbreite der Gruppierungen macht also eine Definition dessen, was in dieser Studie unter Bürgerwehren verstanden werden soll, notwendig. In der Forschungsliteratur existieren unterschiedliche Definitionen zeitgenössischer Bürgerwehren. Thomas Schmidt-Lux beschreibt drei konstante Elemente von Bürgerwehren: »Ihr Charakter als nicht-staatlicher Akteur, der Einsatz oder mindestens die Androhung von Gewalt und schließlich ihr Anspruch auf Gewährleistung von Sicherheit und sozialer Ordnung. ${ }^{17} \mathrm{Er}$ unterscheidet darüber hinaus verschiedene Typen von Bürgerwehren anhand ihres Verhältnisses zum Staat (vgl. Kapitel 5).

Anika Hofmann beschreibt Bürgerwehren aus einer kriminologischen Perspektive. Ihrer Definition nach sind Bürgerwehren »ein in der Regel auf Zeit angelegter Zusammenschluss privater Personen, die in (selbst-)organisierter Form die Über- oder Bewachung eines bestimmten abgegrenzten öffentlichen Raumes durch demonstrative Wachsamkeit zum Zwecke des (präventiven) Schutzes vor sozial unerwünschtem Verhalten anderer Personen für sich beanspruchen oder übernehmen. $\aleph^{18}$

15 Vgl. Schmidt-Lux, Thomas (2018): Bürgerwehren als kollektive Akteure im Feld von Sicherheit und Recht. In: ZeFKo 7 Jg. (2018), Heft 1, S. 141.

Vgl. Hoffmann, Anika (2019): Bürgerwehren und ihre Bedeutung im öffentlichen Raum. Abweichendes Verhalten als kriminologisch relevantes Phänomen. Wiesbaden: VS Verlag. S. 58 und 63.

17 Vgl. Schmidt-Lux, Thomas (2018): Bürgerwehren als kollektive Akteure im Feld von Sicherheit und Recht. In: ZeFKo 7 Jg. (2018), Heft 1, S. 134.

18 Vgl. Hoffmann, Anika (2019): Bürgerwehren und ihre Bedeutung im öffentlichen Raum. Abweichendes Verhalten als kriminologisch relevantes Phänomen. Wiesbaden: VS Verlag. S. 34. 
Diese Definition erfasst das Phänomen zeitgenössischer Bürgerwehren sehr präzise. Jedoch enthält es keine inhaltliche Bestimmung der Motivationen der Mitglieder für das Auftreten als Bürgerwehr. Diese sind jedoch zentral, um Bürgerwehren von anderen Phänomenen privater Selbsthilfe zu unterscheiden.

So stellen beispielsweise auch Antifa-Gruppen das Gewaltmonopol des Staates infrage. Aber die Präsenz lokal agierender Antifa-Gruppen im öffentlichen Raum zielt nicht darauf ab, die eigenen Privilegien zu sichern; vielmehr sollen dieselben Privilegien auch für von Rassismus betroffene Personen gelten - für Personen, die sich in sogenannten »National befreiten Zonen«, in denen rechtsextreme Gruppen den öffentlichen Raum kontrollieren, nicht frei bewegen können.

»Antifa-Praxis (zielt) darauf ab, ähnlich verfassten Akteuren - Angehörigen rechter Straßenkulturen, Neonazis, aber auch Initiativen gegen Flüchtlingsheime-Grenzen zu setzen, ihnen den öffentlichen Raum streitig zu machen und Druck auf sie aufzubauen. ${ }^{19}$

Nils Schuhmacher verweist hier auf die Unterscheidung zwischen Militanz und Gewalt. Bei Antifa-Gruppen gehört zwar ein militantes Auftreten, im Sinne einer kämpferischen Grundhaltung, zur Identität. Gegen Personen gerichtete Gewalt stellt aber kein notwendiges Mittel dar und ist kein Identitätsmerkmal. ${ }^{20}$

In diesem Zusammenhang ist außerdem die Argumentation von Christoph Butterwege relevant. Demnach verkenne eine Gleichsetzung rechtsund linksextremistischer Gruppen, dass die Gefahr für die parlamentarische Demokratie häufiger von den Eliten ausgehe als von den politischen Rändern. Die Gleichsetzung von inhaltlich völlig unterschiedlichen Akteursgruppen ignoriere darüber hinaus die Motive und Interessen ihrer Mitglieder, die jedoch zentral seien für das Verständnis der Gruppen. ${ }^{21}$

Bürgerwehren stellen das Gewaltmonopol des Staates infrage. Trotzdem stabilisieren sie im Ergebnis die Machtverhältnisse zwischen den sozialen

19 Schuhmacher, Nils (2017): »Küsst die Faschisten«. Autonomer Antifaschismus als Begriff und Programm. In: (Anti-)Faschismus. APuZ 42-43/2017. S. 39.

20 Ebd., S. 40.

21 Vgl. Butterwegge, Christoph (2011): Linksextremismus = Rechtsextremismus? Über die Konsequenzen einer falschen Gleichsetzung. In: Birsl, Ursula (Hg.) (2011): Rechtsextremismus und Gender. Opladen: Budrich. S. $39 f$. 
Gruppen innerhalb der Gesellschaft. Hinter dem Auftreten als Bürgerwehr steht der Versuch, eigene Privilegien zu verteidigen. ${ }^{22}$ Um eine klare Definition von Bürgerwehren zu erhalten, wird hier daher die Definition von Hoffmann um die Motivationen der Mitglieder für ein Auftreten als Bürgerwehr ergänzt. Daraus ergibt sich nun folgende Definition für Bürgerwehren:23

Eine Bürgerwehr ist ein Zusammenschluss privater Personen, die auf eine wahrgenommene Unsicherheit reagieren und im öffentlichen Raum demonstrativ Präsenz zeigen, um deviates Verhalten anderer Bürger*innen zu sanktionieren und eigene Privilegien zu verteidigen.

Das Verhältnis zum Staat, das in der Definition nach Schmidt-Lux so zentral ist, findet hier nur indirekt Eingang in die Definition. So sind die Mitglieder der Bürgerwehren private Personen, also keine staatlichen Akteure. Darüber hinaus sind wahrgenommene Unsicherheiten als Grundlage für die Gründung von Bürgerwehren Teil der Definition. Bürgerwehren orientieren sich nicht an den materiellen Indikatoren von Sicherheit; eine geringe Polizeipräsenz oder ein Fehlen staatlicher Strukturen reicht nicht für eine qualifizierte Definition aus, wie insbesondere die in dieser Studie untersuchte Bürgerwehr in Berlin Mitte zeigt.

Schließlich klammert diese Definition Sicherheitspartnerschaften und andere Formen polizeilich organisierten Bürgerengagements für Sicherheit aus. Denn diese Gruppen unterstehen in ihrer Funktion als Sicherheitsakteure dem Staat, sie sind an seine Anweisungen gebunden und sind keine Privatpersonen, die sich eigenmächtig für die Sicherheit zusammenschließen.

22 Auch Matthias Quent verweist in seiner Studie darauf, dass Bürgerwehren ein Projekt zu Verteidigung von eigenen Privilegien sind. Vgl. Quent, Matthias (2016): Bürgerwehren. Hilfssheriffs oder inszenierte Provokation? Amadeus Antonio Stiftung. Cottbus: Druckzone. S. $12 f$.

23 Der Begriff Bürgerwehr wird in dieser Studie als wissenschaftliche Kategorie verwendet. Demnach werden alle Gruppen als Bürgerwehren bezeichnet, die den Kriterien dieser Definition genügen. Abweichende Selbstbezeichnungen beispielsweise als »Bürgerstreife« werden in Anführungszeichen gesetzt, da sie mitunter Strategien der Verharmlosung der Akteure darstellen. 


\subsubsection{Bürgerwehrähnliche Phänomene in anderen Ländern}

Das Phänomen der Bürgerwehren ist keineswegs auf Deutschland beschränkt. Sie lassen sich fast überall finden: In den USA, in Russland und insbesondere auch in Staaten mit schwachem staatlichen Gewaltmonopol, so zum Beispiel gegenwärtig in Mexiko. ${ }^{24}$ Auch gründen sich in europäischen Staaten als Reaktion auf die jüngsten Migrationsbewegungen verstärkt rechte Bürgerwehren. ${ }^{25}$

Die internationale Forschungsdebatte bezieht sich entweder auf Vigilantismus in Räumen begrenzter Staatlichkeit oder auf den US-amerikanischen Kontext. ${ }^{26}$ Einen guten Überblick über Bürgerwehr-ähnliche Bewegungen auf der ganzen Welt gibt der Band »Global Vigilantes«, herausgegeben von David Pratten und Atreyee Sen. ${ }^{27}$ Die Beiträge des Bandes zeigen eine enorme Bandbreite vigilanter Aktivitäten auf der ganzen Welt. Die jeweiligen sozialen und politischen Hintergründe, die zum Auftreten von Vigilantismus führen, variieren zwischen den einzelnen Ländern ebenso wie die Funktion, die vigilante Gruppen in der lokalen Bevölkerung spielen. Das macht vergleichende Studien der Phänomene in den einzelnen Ländern schwierig, was auch die geringe

24 Vgl. Coen, Amrai/Brennecke, Fabian (2015): Die Zeugen von Iguala. Online unter: www .zeit.de/feature/mexiko-iguala-studenten-mord-buergerwehr (Zugriff am 2.1.2021).

25 So zum Beispiel in Finnland. Vgl. Wolff, Reinhard (2016): Soldiers of Odin auf Patrouille. In: Die tageszeitung vom 18. 01. 2016. Online unter: http://taz.de/Buergerwehren-in-Fi nnland/!5266411/ (Zugriff am 2.1.2021).

26 Vgl. bspw. Chojnacki, Sven/Branović, Željko (2007): Privatisierung von Sicherheit? Formen von Sicherheits-Governance in Räumen begrenzter Staatlichkeit. In: Sicherheit und Frieden. Vol. 25, Nr. 4, Themenschwerpunkt: »Privatisierung« von Sicherheit. S. 163-169. Sowie: Schneckener, Ulrich (2015): Status-quo-orientierte Gewalt? Zur Charakterisierung von Milizen. In: Sicherheit und Frieden/Security and Peace, 33(4), S. 173-179. Sowie: Pratten, David und Sen, Atreyee (2008): Global Vigilantes. New York: Columbia University Press. Und Richard Maxwell Brown beschreibt für den USamerikanischen Kontext einen Neovigilantismus. Dieser unterscheidet sich vom klassischen Vigilantismus dadurch, dass seine Haupttätigkeit in Patrouillenaktionen zum Zwecke der Aufdeckung und Berichterstattung besteht. Außerdem arbeiteten neovigilante Gruppen häufig mit der Polizei zusammen. Vgl. Brown, Richard Maxwell (1975): Strain of Violence. Historical Studies of American Violence and Vigilantism. New York: Oxford University Press. S. 127-129.

27 Vgl. Pratten, David/Sen, Atreyee (2008): Clobal Vigilantes. New York: Columbia University Press. 
Anzahl international vergleichender Analysen zu erklären vermag. Ray Abrahams plädiert daher für eine Verbindung der Herangehensweisen. ${ }^{28}$ Denn Vigilanten agieren mittlerweile über nationale Grenzen hinweg: Die Bürgerwehren an der US-amerikanisch-mexikanischen Grenze beispielsweise wirken mit ihren Aktionen sowohl auf die mexikanische als auch auf die USamerikanische Gesellschaft und Politik. ${ }^{29}$ Roxanne Doty analysiert diese Civilian Border Patrols mithilfe der Copenhagen School of Security und beschreibt Grenzpatrouillen durch Bürger*innen als inhärent globales Phänomen:

»Border vigilantes like the Minutemen make it clear that this is not simply a local phenome non, but rather is inextricably linked to the global. ${ }^{30}$

\subsection{Bürgerwehren als interdisziplinärer Forschungsgegenstand}

Nach den Ereignissen in der Silvesternacht 2015/16 am Kölner Hauptbahnhof und der darauffolgenden medialen Debatte gründeten sich in ganz Deutschland Bürgerwehren. Wie viele genau, ist schwer zu sagen, aber alleine in Niedersachsen gründeten sich 30 Bürgerwehren innerhalb weniger Wochen. ${ }^{31}$ Die Google-Suchanfragen zu Bürgerwehren schnellten in die Höhe (siehe Abbildung 1).

28 Vgl. Abrahams, Ray: Some thoughts on the comparative study of vigilantism. In: Pratten, David und Sen, Atreyee (2008): Clobal Vigilantes. New York: Columbia University Press. S. 419-442.

29 Vgl. Doty, Roxanne Lynn (2007): States of Exception on the Mexico-U.S. Border: Security, »Decisions«, and Civilian Border Patrols. In: International Political Sociology 2007: 1. S. 113-137.

30 Vgl. ebd., S. 118. Vgl. zu den minuteman außerdem: Akers Chacón, Justin/Davis, Mike (2007): Crossing the Border. Migration und Klassenkampf in der US- amerikanischen Ceschichte. Berlin: Assoziation A. Sowie: Chavez, Leo R. (2008): Spectacle in the desert: the Minuteman project on the US-Mexico border. In: Pratten, David (Hg.): Clobal Vigilantes. New York: Columbia University Press.

31 So Niedersachsens Innenminister Boris Pistorius (SPD): Vgl. Göttinger Tageblatt Online vom 18.2.2016: https://www.goettinger-tageblatt.de/Nachrichten/Der-Norden/ Niedersachsens-Sicherheitsbehoerden-haben-31-Buergerwehren-im-Blick (Zugriff am 2.1.2021). 
Abbildung 1: Google-Suchanfragen zum Begriff »Bürgerwehren«

Interesse im zeitlichen Verlauf

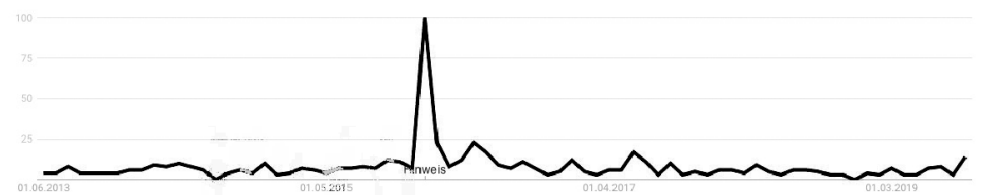

Quelle: Googletrends: https://trends.google.de/trends/explore?date $=2013-07-01 \% 202020$ -12-15\&geo $=\mathrm{DE} \& \mathrm{q}=\mathrm{B} \% \mathrm{C} 3 \% \mathrm{BCrgerwehren}$ (Zugriff am 16.12.2020)

Trotz der zahlreichen Neugründungen seit 2016 und der medialen Aufmerksamkeit gibt es relativ wenige wissenschaftliche Beiträge zum Thema. ${ }^{32}$ Da Bürgerwehren ein interdisziplinärer Forschungsgegenstand sind, wird im folgenden Überblick neben politikwissenschaftlichen Quellen auch auf Beiträge aus der Soziologie, Kriminologie und den Kulturwissenschaften Bezug genommen.

Bürgerwehren werden in der Forschungsliteratur auf zwei Arten eingeordnet: Einerseits im Rahmen der zivilen Sicherheitsforschung als community policing, andererseits in staatstheoretischen Debatten als Phänomen des Vigilantismus. Diese beiden Forschungsdebatten werden im Folgenden überblicksartig dargestellt. Anschließend wird dargelegt, wie sie mit Bezug auf den Forschungsgegenstand verschränkt werden können.

32 Vgl. Hoffmann, Anika (2019): Bürgerwehren und ihre Bedeutung im öffentlichen Raum. Abweichendes Verhalten als kriminologisch relevantes Phänomen. Wiesbaden: VS Verlag. S. 12. 


\subsubsection{Zum Stand der Zivilen Sicherheitsforschung}

Sicherheit ist ein menschliches Grundbedürfnis. ${ }^{33}$ Sicherheitsgefühle sind jedoch kein Ausdruck objektiv vergleichbarer materieller Sicherheitslagen, ${ }^{34}$ sie sind ein subjektives Abwägen einer Beherrschbarkeit von kalkulierbaren Risiken..$^{35}$ Diese Wahrnehmung von Risiken und unser Begriff von Sicherheit sind moderne Erscheinungen. Bevor sich Menschen als die Geschicke der Welt beeinflussende Wesen begriffen, war Sicherheit keine Größe, die es zu maximieren galt. Erst in der Moderne wurde Sicherheit zu einem Problem, für das Lösungen gefunden werden mussten. Dem modernen Nationalstaat kommt dabei die Rolle des Sicherheitsgaranten zu: ${ }^{36}$ Er hat die Aufgabe, Rahmenbedingungen zu schaffen, die es den Bürger*innen ermöglichen, sich sicher zu fühlen. ${ }^{37}$ Sicherheit ist zu einem Wert geworden. ${ }^{38}$ Und die Performanz des Staates wird an seiner Fähigkeit, Sicherheit zu gewährleisten, gemessen. Sicherheit ist zum »Goldstandard des Politischen « geworden. ${ }^{39}$

Mit zunehmendem technologischem Fortschritt und einer komplexeren Organisation von Gesellschaften veränderten sich die Risiken, sie verloren ihre Kalkulierbarkeit und Beherrschbarkeit. Damit verändern sich auch die

Vgl. Maslow, Abraham (1977): Motivation und Persönlichkeit. Olten/Freiburg: Walter. S. 79.

34 Vgl. Albrecht, Hans-Jörg (2010): Neue Bedrohungen? Wandel von Sicherheit und Sicherheitserwartungen. In: Zoche/Kaufmann/Haverkamp (Hg.) (2010): Zivile Sicherheit. Gesellschaftliche Dimensionen gegenwärtiger Sicherheitspolitiken. Bielefeld: transcript. S. 118 sowie S. 119, Abbildung 10.

Vgl. Kaufmann, Franz-Xaver (2003): Sicherheit. Das Leitbild beherrschbarer Komplexität. In: Lessenich, Stephan (Hg.) (2003): Wohlfahrtsstaatliche Grundbegriffe. Historische und aktuelle Diskurse. Frankfurt a.M.: Campus. S. 91.

Zurück gehen diese Argumentationen auf Hobbes, Thomas (1651): Leviathan. Hamburg (1996): Meiner.

37 Vgl. Albrecht, Hans-Jörg (2010): Neue Bedrohungen? Wandel von Sicherheit und Sicherheitserwartungen. In: Zoche/Kaufmann/Haverkamp (Hg.) (2010): Zivile Sicherheit. Gesellschaftliche Dimensionen gegenwärtiger Sicherheitspolitiken. Bielefeld: transcript. S. 112.

38 Kaufmann, Franz-Xaver (1973): Sicherheit als soziologisches und sozialpolitisches Problem. 2. umgearbeitete Auflage, Stuttgart: Ferdinand Enke Verlag. S. 28ff.

39 Vgl. Daase, Christopher (2010): Der Wandel der Sicherheitskultur. Ursachen und Folgen des erweiterten Sicherheitsbegriffs. In: Zoche/Kaufmann/Haverkamp (Hg.) (2010): Zivile Sicherheit. Gesellschaftliche Dimensionen gegenwärtiger Sicherheitspolitiken. Bielefeld: transcript. S. 139. 
Anforderungen an den Staat, Sicherheit zu schaffen. Wolfgang Bonß unterscheidet in Anlehnung an Ulrich Beck ${ }^{40}$ "alte « von "neuen« Risiken. "Alte« Risiken sind demnach kalkulierbar und in ihren Schadenserwartungen begrenzt, während »neue« Risiken hypothetisch bleiben, nicht vollständig bekannt sind und in ihren Schäden durch Geld nicht zu kompensieren sind. Beispiele für »neue« Risiken sind Kernkraftwerke oder Gentechnologie. Aus der Unkalkulierbarkeit »neuer« Risiken folgt eine Angst vor Gefahr, die die Risikofreudigkeit angesichts »alter « Risiken ersetzt. ${ }^{41}$ Das Forschungsgebiet »Zivile Sicherheit« bildet diesen Wandel ab.

»Cleich ob man terroristische oder kriminelle Bedrohungen, großtechnische Unfälle oder durch Naturereignisse hervorgerufene Katastrophen adressiert: Im Zeichen ziviler Sicherheit werden all diese Gefährdungen auf ein grundlegendes Problem zurückgeführt - nämlich auf die Verwundbarkeit des modernen Lebens. $\ll^{42}$

Weil staatliche Sicherheitsstrategien auf vielschichtige Bedrohungsszenarien reagieren müssen, geht es in komplexen Gesellschaften nicht mehr nur um den Schutz einzelner Bürger*innen, sondern auch um die Sicherung von Versorgungs-, Verkehrs- und Informationsinfrastrukturen. ${ }^{43}$ Der Wandel der Risiken geht mit einem Wandel des (Un-)Sicherheitsbewusstsein der Bürger*innen einher. ${ }^{44}$ Die zivile Sicherheitsforschung untersucht die Folgen dieses Wandels sowie die staatlichen und gesellschaftlichen Antworten auf neue Sicherheitsherausforderungen..$^{45}$

40 Vgl. Beck, Ulrich (1986): Risikogesellschaft. Auf dem Weg in eine andere Moderne. Frankfurt a.M.: Suhrkamp.

41 Vgl. Bonß, Wolfgang (2010): (Un-)Sicherheit in der Moderne. In: Zoche/Kaufmann/Haverkamp (Hg.) (2010): Zivile Sicherheit. Cesellschaftliche Dimensionen gegenwärtiger Sicherheitspolitiken. Bielefeld: transcript. S. 62.

42 Zoche/Kaufmann/Haverkamp (Hg.) (2010): Zivile Sicherheit. Cesellschaftliche Dimensionen gegenwärtiger Sicherheitspolitiken. Bielefeld: transcript. S. 9.

43 Ebd., S. 9.

44 Vgl. bspw. Zoche/Kaufmann/Haverkamp (Hg.) (2010): Zivile Sicherheit. Gesellschaftliche Dimensionen gegenwärtiger Sicherheitspolitiken. Bielefeld: transcript. (Insbes. die Beiträge von Hans-Jörg Albrecht, Rudolf Egg, Christopher Daase, Wolfgang Bonß, und Dietrich Dörner). Sowie: Baban, Constance Pary (2013): Der innenpolitische Sicherheitsdiskurs in Deutschland: zur diskursiven Konstruktion des sicherheitspolitischen Wandels 2001-2009. Konstanz: VS Verlag.

45 Einen guten Überblick über den Stand der Forschung bieten die Sammelbände der Reihe »Zivile Sicherheit. Schriften zum Fachdialog Sicherheitsforschung« im LIT Ver- 
Um einen Überblick über den Stand der zivilen Sicherheitsforschung zu geben, wird zunächst der Wandel der (Un-)Sicherheitswahrnehmungen von Bürgerinnen und Bürgern beschrieben. Anschließend wird der Stand der Debatte bezüglich der gesellschaftlichen und institutionellen Folgen dieses Wandels dargelegt. Hier sind auf gesellschaftlicher Ebene insbesondere ein Wandel der Sicherheitskultur zu nennen sowie auf institutioneller Ebene eine Privatisierung von Sicherheit. Auch wenn es bisher kaum Studien gibt, die das Phänomen Bürgerwehren im Kontext ziviler Sicherheit analysieren, so ist dieses Forschungsfeld zentral für eine Verortung des Forschungsgegenstandes in der wissenschaftlichen Debatte. Bürgerwehren reagieren auf Unsicherheitsgefühle und lassen sich als Phänomen privater Sicherheit begreifen. Außerdem finden Bürgerwehren als Form des Community Policing am Rande Eingang in die Debatte - diese Studien beziehen sich jedoch auf ältere Beispiele und berücksichtigen nicht die aktuellen Gründungen seit 2016.

\subsubsection{1 (Un-)Sicherheitswahrnehmungen im Wandel}

Der Beschreibung von Unsicherheitswahrnehmungen liegt die These zugrunde, dass sich eine gefühlte Sicherheit der Bürger*innen von der tatsächlichen Viktimisierungswahrscheinlichkeit unterscheidet. ${ }^{46}$ Generell ist eine Unterscheidung zwischen »objektiver« Unsicherheit und gefühlter Unsicherheit irreführend, suggeriert sie doch eine objektive Messbarkeit von Sicherheit. ${ }^{47}$ Natürlich muss auf den materiellen Gehalt von Unsicherheitswahrnehmungen verwiesen werden, doch bei der Beschreibung von materiellen Grund-

lag. Vgl. Zoche/Kaufmann/Haverkamp (Hg.) (2010): Zivile Sicherheit. Cesellschaftliche Dimensionen gegenwärtiger Sicherheitspolitiken. Bielefeld: Trascript. Sowie: Hoch, Hans/Zoche, Peter (Hg.) (2014): Sicherheiten und Unsicherheiten. Berlin: LIT Verlag. Und: Zoche, Peter et.al. (2016): Grenzenlose Sicherheit? Gesellschaftliche Dimensionen der Sicherheitsforschung. Berlin: LIT Verlag. Sowie: Ellebrecht/Kaufmann/Zoche (2019): (Un-)Sicherheiten im Wandel. Gesellschaftliche Dimensionen von Sicherheit. Berlin: LIT Verlag.

46 Das zeigt beispielsweise die in den 1960er Jahren entstandene Viktimisierungsforschung, die die Kriminalitätsbelastung sowie die Einschätzung der öffentlichen Sicherheit durch die Bevölkerung erfasst und vergleicht. Vgl. hierzu: Albrecht, Hans-Jörg (2014): Sicherheit, Sicherheitsmonitoring und Viktimisierungsstudien - Ansätze und Ergebnisse. In: Hoch, Hans/Zoche, Peter (Hg.) (2014): Sicherheiten und Unsicherheiten. Berlin: LIT Verlag. S. 80.

47 Vgl. zur Messbarkeit von Sicherheit: Armborst, Andreas (2014): Sicherheitsforschung. In: Hoch, Hans/Zoche, Peter (Hg.) (2014): Sicherheiten und Unsicherheiten. Berlin: LIT Verlag. S. 80 sowie S. $37 f f$. 
lagen von (Un-)Sicherheitsgefühlen wird auf Daten verwiesen, die ihrerseits durch die Brille kulturell geprägter Bedrohungsvorstellungen betrachtet werden. Ronald Hitzler spricht daher von einer Inszenierung der Inneren Sicherheit, in der Bedrohung und Bedrohungsbewältigung ständig (re)konstruiert werden. ${ }^{48}$

Im subjektiven Sicherheitsempfinden werden Ängste psychologisch verarbeitet, und das geschieht nicht immer rational. Hirtenlehner zeigt beispielsweise in seinen Studien eine projektile Transformation von sozialen Ängsten, etwa von der Angst vor Arbeitslosigkeit in Kriminalitätsfurcht. ${ }^{49}$ Und Albert Scherr beschreibt eine Transformation von sozialen Ängsten in eine Angst vor unkontrollierter Einwanderung. ${ }^{50}$ Wacquant spricht in diesem Zusammenhang mit Bezug auf die USA von einem Übergang vom Sozialstaat zum Strafstaat. ${ }^{51}$

Die Auswirkungen neuer Bedrohungsszenarien lassen sich exemplarisch am Beispiel des internationalen Terrorismus beschreiben. Die Anschläge vom 11. September 2001 stellen diesbezüglich eine Zäsur dar. Die auch in Europa präsente Bedrohung durch den internationalen Terrorismus hat die Wahrnehmung von Sicherheit grundlegend verändert. So zeigt Baran in seiner Untersuchung des sicherheitspolitischen Diskurses 2001-2009, ${ }^{52}$ dass das vermeintlich hohe Sicherheitsbedürfnis in der Bevölkerung angesichts neuer Bedrohungslagen wie dem internationalen Terrorismus zu einer Verflechtung

48 Vgl. hierzu: Hitzler, Ronald (1998): Bedrohung und Bewältigung. Einige handlungstheoretisch triviale Bemerkungen zur Inszenierung >Innere Sicherheit, in: Hitzler, Ronald/Peters, Helge (Hg.): Inszenierung: Innere Sicherheit. Daten und Diskurse. Opladen: Leske + Budrich. S. 203-212.

49 Vgl. hierzu: Hirtenlehner, Helmut (2006): Kriminalitätsfurcht - Ausdruck generalisierter Ängste und schwindender Gewissheiten? In: KZfSS Kölner Zeitschrift für Soziologie und Sozialpsychologie. Januar 2006, Vol. 58, Issue 2. S. 307-331. Sowie: Scherr, Albert (2014): Kriminalität, innere Sicherheit und soziale Unsicherheit. Sicherheitsdiskurse als Bearbeitung gesellschaftsstrukturell bedingter Ängste. Wiesbaden: VS Verlag.

50 Vgl. Scherr, Albert (2014): Kriminalität, innere Sicherheit und soziale Unsicherheit: Sicherheitsdiskurse als Bearbeitung gesellschaftsstrukturell bedingter Ängste. Wiesbaden: VS Verlag. S. 3.

51 Vgl. Wacquant, Loïc (2008): Die Bedeutung des Cefängnisses für das neue Armutsregime. In: PROKLA. Zeitschrift für Kritische Sozialwissenschaft, 38(152). S. 399-412.

52 Baban, Constance Pary (2013): Der innenpolitische Sicherheitsdiskurs in Deutschland. Zur diskursiven Konstruktion des sicherheitspolitischen Wandels 2001-2009. Konstanz: VS Verlag. Insbes. S. 341-348. 
von innerer und äußerer Sicherheit geführt hat und eine Normalisierung präventiver Sicherheitspolitiken ${ }^{53}$ ermöglichte. ${ }^{54}$

Die Auseinanderentwicklung von materiellen Sicherheitslagen und subjektiven Sicherheitsgefühlen am Beispiel des internationalen Terrorismus zeigt: Nicht die Unsicherheit ist größer geworden, sondern die subjektive Viktimisierungsangst. ${ }^{55}$ Und so beziehen sich einige Maßnahmen der Inneren Sicherheit, wie beispielsweise mehr Polizeipräsenz im öffentlichen Raum, stärker auf die Reduktion von Angst als auf die Vermeidung von Kriminalität selbst. ${ }^{56}$ Ein Beispiel hierfür ist die Ausrüstung von Polizeikräften mit Maschinenpistolen auf deutschen Weihnachtsmärkten im Anschluss an den Anschlag auf den Weihnachtsmarkt am Berliner Breitscheidplatz 2016.

Wie sicher sich die Bevölkerung fühlt, hängt also auch davon $a b$, wie der Staat Sicherheitskommunikation ${ }^{57}$ organisiert. Zwar spielt die Angst vor Kriminalverbrechen in Deutschland im Vergleich zu anderen Ängsten (Krankheit, Arbeitslosigkeit) eine eher untergeordnete Rolle. ${ }^{58}$ Studien bestätigen jedoch einen Einfluss der Wahrnehmung des Wohnumfelds auf die Kriminalitätsfurcht der Bürger*innen. ${ }^{59}$ Hier sind es gerade Polizeistreifen, die ein Ge-

53 Vgl. hierzu auch die Studie von Matthias Schulze zur sprachlichen Konstruktion von Sicherheit und Unsicherheit am Beispiel der Debatten um Vorratsdatenspeicherung und Online-Durchsuchungen: Schulze, Matthias (2012): Die Sprache der (Un-)Sicherheit. Die Konstruktion von Bedrohung im sicherheitspolitischen Diskurs der Bundesrepublik Deutschland. Band 51, Marburg: Tectum Verlag.

So auch: Heinrich, Stephan/Lange, Hans-Jürgen (2008): Erweiterung des Sicherheitsbegriffs. In: Lange/Ohly/Reichertz (Hg.) (2008): Auf der Suche nach neuer Sicherheit. Wiesbaden: VS Verlag. S. 265.

55 Vgl. Priester, Karin (2010): Innere Sicherheit und Bürgerwehren als Politikum. In: Neue Gesellschaft, Frankfurter Hefte, Nr. 12/2010. S. 34.

Vgl. Reuband, Karl-Heinz (2008): Kriminalitätsfurcht. Erscheinungsformen, Trends und soziale Determinanten. In: Lange/Ohly/Reichertz (Hg.) (2008): Aufder Suche nach neuer Sicherheit. Wiesbaden: VS Verlag. S. 233.

57 Vgl. hierzu: Schirmer, Werner (2018): Bedrohungskommunikation. Eine gesellschaftstheoretische Studie zu Sicherheit und Unsicherheit. Wiesbaden: VS Verlag.

Vgl. Egg, Rudolf (2010): Kriminalität: Furcht und Realität. In: Zoche/Kaufmann/Haverkamp (Hg.) (2010): Zivile Sicherheit. Cesellschaftliche Dimensionen gegenwärtiger Sicherheitspolitiken. Bielefeld: transcript. S. 131.

59 Vgl. Albrecht, Hans-Jörg (2010): Neue Bedrohungen? Wandel von Sicherheit und Sicherheitserwartungen. In: Zoche/Kaufmann/Haverkamp (Hg.) (2010): Zivile Sicherheit. Gesellschaftliche Dimensionen gegenwärtiger Sicherheitspolitiken. Bielefeld: transcript. S. 121ff. Sowie Hirtenlehner, Helmut (2006): Kriminalitätsfurcht-Ausdruck generalisierter Ängste und schwindender Cewissheiten? In: KZfSS Kölner Zeitschrift 
fühl von Sicherheit vermitteln, ohne direkt zur Kriminalitätsreduktion beizutragen. In ihrer kriminologischen Analyse von Bürgerwehren im öffentlichen Raum beschreibt Annika Hoffmann Bürgerwehren als Ausdruck gescheiterter staatlicher Sicherheitskommunikation. ${ }^{60}$

Christopher Dase allerdings weist darauf hin, dass bei dem allgemein konstatierten Wandel des Sicherheitsempfindens durch neue Bedrohungen diese neuen Risiken gar nicht so neu sind, sondern vielmehr Ausdruck einer gewandelten Wahrnehmung politischer Probleme. ${ }^{61}$ Dieses Phänomen wird in der Forschungsdebatte als »Wandel der Sicherheitskultur« verhandelt.

\subsubsection{Sicherheitskultur in Deutschland}

Sicherheitskultur bezeichnet die kulturelle Praxis, mit der in einer Gesellschaft Sicherheit produziert wird. ${ }^{62}$ Sicherheitskulturen, die die Abwesenheit von Unsicherheit als zu erstrebendes Ideal proklamieren, führen zu Stillstand und Regression. Anders wird eine Gesellschaft agieren, deren Sicherheitskultur - in dem Wissen, dass absolute Sicherheit nie möglich ist - ein bestimmtes Level an Unsicherheit akzeptiert. Herfried Münkler spricht hier von »Welten der Sicherheit « und »Kulturen des Risikos «. ${ }^{63}$

für Soziologie und Sozialpsychologie. Januar 2006, Vol. 58, Issue 2. S. 307-331. Sowie Lüdemann, Christian (2006): Kriminalitätsfurcht im urbanen Raum. Eine Mehrebenenanalyse zu individuellen und sozialräumlichen Determinanten verschiedener Dimensionen von Kriminalitätsfurcht. In: KZfSS Kölner Zeitschrift für Soziologie und Sozialpsychologie Januar 2006, Vol. 58, Nr. 2. S. 285-306.

60 Vgl. Hoffmann, Anika (2019): Bürgerwehren und ihre Bedeutung im öffentlichen Raum. Abweichendes Verhalten als kriminologisch relevantes Phänomen. Wiesbaden: VS Verlag. S. 150.

61 Vgl. Daase, Christopher (2010): Der Wandel der Sicherheitskultur. Ursachen und Folgen des erweiterten Sicherheitsbegriffs. In: Zoche/Kaufmann/Haverkamp (Hg.) (2010): Zivile Sicherheit. Gesellschaftliche Dimensionen gegenwärtiger Sicherheitspolitiken. Bielefeld: transcript. S. 140.

62 Vgl. Wendekamm, Michaela/Terberl, Christina (2014): Un/Sicherheit als Un/Sichtbarkeit. Sicherheitskultur als Sichtbarkeitskultur. In: Lange, Hans-Jürgen/Wendekamm, Michaela/Endreß, Christian (Hg.) (2014): Dimensionen der Sicherheitskultur. Wiesbaden: VS Verlag. S. 323.

63 Vgl. Münkler, Herfried (2010): Strategien der Sicherung: Welten der Sicherheit und Kulturen des Risikos. Theoretische Perspektiven. In: Münkler/Bohlender/Meurer (Hg.): Sicherheit und Risiko. Über den Umgang mit Gefahr im 21. Jahrhundert. Bielefeld: transcript. S. 11-34. 
In komplexen Gesellschaften mit neuen Bedrohungslagen wird die kulturelle Praxis der Sicherheitsherstellung davon geprägt, dass sich die Bedrohungen nicht mehr in Ursache und Wirkung aufschlüsseln lassen. Stattdessen spricht man in der Sicherheitsforschung von »vernetzten Bedrohungsfeldern ${ }^{64}{ }^{64}$ Diese stellen die Sicherheitspolitik von Staaten zunehmend vor Aufgaben, die sie nicht lösen kann, ${ }^{65}$ und es geraten Gefahren in den Blick, die noch keine sind. Diese präventive Wende lässt Anforderungen entstehen, proaktiv tätig zu werden. ${ }^{66}$ Zudem führt die »Globalisierung der Staatlichkeit« zu einem Verlust staatlicher Steuerungsfähigkeit, die sich im Sicherheitsdiskurs ablesen lässt. Gegen organisierte Kriminalität oder internationalen Terrorismus können einzelne Staaten kaum alleine etwas ausrichten. ${ }^{67}$

Christopher Daase beschreibt diesen Wandel der Sicherheitskultur in den letzten 50 Jahren und erkennt eine Denationalisierung des Sicherheitsbegriffs. Gesellschaftliche Gefahrenwahrnehmungen emanzipieren sich von staatlichen Sicherheitsbedürfnissen und fordern eine "proaktive Präventionspolitik und Daseinsvorsorge ${ }^{68}$ - beispielsweise durch eine Intensivierung der sozialen Kontrolle im öffentlichen Raum durch Videoüberwachung. ${ }^{69}$

64 Vgl. Endreß, Christian/Heißt, Martin (2014): Von der Sicherheit zur Sicherheitskultur. Über den Umgang mit Komplexität im Sicherheitsdiskurs. In: Lange, HansJürgen/Wendekamm, Michaela/Endreß, Christian (Hg.) (2014): Dimensionen der Sicherheitskultur. Wiesbaden: VS Verlag. S. $19 \mathrm{ff}$.

65 Vgl. Daase, Christopher (2010): Der Wandel der Sicherheitskultur. Ursachen und Folgen des erweiterten Sicherheitsbegriffs. In: Zoche/Kaufmann/Haverkamp (Hg.) (2010): Zivile Sicherheit. Gesellschaftliche Dimensionen gegenwärtiger Sicherheitspolitiken. Bielefeld: transcript. S. 141.

66 Vgl. Heinrich, Stephan/Lange, Hans-Jürgen (2008): Erweiterung des Sicherheitsbegriffs. In: Lange/Ohly/Reichertz (Hg.) (2008): Auf der Suche nach neuer Sicherheit. Wiesbaden: VS Verlag. S. 265.

67 Vgl. Lange, Hans-Jürgen (2010): Zum Wandel der Institutionen und Steuerungsformen des staatlichen Sicherheitssystems. In: Zoche/Kaufmann/Haverkamp (Hg.) (2010): Zivile Sicherheit. Gesellschaftliche Dimensionen gegenwärtiger Sicherheitspolitiken. Bielefeld: transcript. S. 319.

68 Vgl. Daase, Christopher (2010): Der Wandel der Sicherheitskultur. Ursachen und Folgen des erweiterten Sicherheitsbegriffs. In: Zoche/Kaufmann/Haverkamp (Hg.) (2010): Zivile Sicherheit. Gesellschaftliche Dimensionen gegenwärtiger Sicherheitspolitiken. Bielefeld: transcript. S. 155.

69 Vgl. hierzu: Eifler, Stefanie (2008): Soziale Kontrolle im öffentlichen Raum. In: Lange/Ohly/Reichertz (Hg.) (2008): Auf der Suche nach neuer Sicherheit. Wiesbaden: VS Verlag. S. 269-280. 
Dieser Wandel hat zahlreiche Folgen, die in der Forschungsdebatte durchaus kritisch diskutiert werden. Zunächst bringt dieses Setting eine Erweiterung des Sicherheitsbegriffs ${ }^{70}$ mit sich, der ein größeres Potential in sich birgt, politisierte Themen zu Sicherheitsthemen zu machen und somit außerordentliche Maßnahmen zu rechtfertigen ${ }^{71}$ (vgl. hierzu auch Kapitel 2). Diese Entwicklung wird in der Literatur kontrovers betrachtet. Einerseits müssen sich mit dem Aufkommen neuer Technologien auch die Befugnisse von Sicherheitsorganen ändern. ${ }^{72}$ Andererseits entsteht durch die Übertragung von Prinzipien eines erweiterten Sicherheitsbegriffs, der außenpolitischen Sicherheitspolitiken entstammt, auf die Innere Sicherheit die Gefahr, dass diese neuen Instrumente und Feindbilder die bisher im Politikfeld Innere Sicherheit vorherrschenden Prinzipien von Rechtsstaatlichkeit untergraben. ${ }^{73}$

Singelstein und Stolle beschreiben in ihrer Kritik der Sicherheitsgesellschaft einen Wandel in der Ausübung von sozialer Kontrolle. Diese werde nicht mehr durch gesellschaftlich geteilte Normen hergestellt, sondern disziplinierend durch die »Verwaltung von Devarianz und einem Management von Risiken. «Die Autoren konstatieren in der Folge einen »Bedeutungsverlust von vormals zentralen gesellschaftlichen Prinzipien wie Demokratie, sozialer Gleichheit, individueller Freiheit und Selbstbestimmung. ${ }^{74}$

Diese Gefahr für den demokratischen Rechtsstaat arbeitet insbesondere der theoretische Ansatz der Versicherheitlichung heraus, der als Analyserah-

70 Vgl. hierzu: Daase, Christopher (2010): Der Wandel der Sicherheitskultur. Ursachen und Folgen des erweiterten Sicherheitsbegriffs. In: Zoche/Kaufmann/Haverkamp (Hg.) (2010): Zivile Sicherheit. Cesellschaftliche Dimensionen gegenwärtiger Sicherheitspolitiken. Bielefeld: transcript. S. 139-158. Sowie: Heinrich, Stephan/Lange, Hans-Jürgen (2008): Erweiterung des Sicherheitsbegriffs. In: Lange/Ohly/Reichertz (Hg.) (2008): Auf der Suche nach neuer Sicherheit. Wiesbaden: VS Verlag. S. 253-268.

71 Vgl. Endreß, Christian/Heißt, Martin (2014): Von der Sicherheit zur Sicherheitskultur. Über den Umgang mit Komplexität im Sicherheitsdiskurs. In: Lange, HansJürgen/Wendekamm, Michaela/Endreß, Christian (Hg.) (2014): Dimensionen der Sicherheitskultur. Wiesbaden: VS Verlag. S. 21.

Vgl. hierzu: Maurer, Jürgen (2013): Sicherheit ohne Angst. In: Daase/Engert/Junk (Hg.) (2013): Verunsicherte Cesellschaft - überforderter Staat. Zum Wandel der Sicherheitskultur. Frankfurt a.M.: Campus.

73 Vgl. Heinrich, Stephan/Lange, Hans-Jürgen (2008): Erweiterung des Sicherheitsbegriffs. In: Lange/Ohly/Reichertz (Hg.) (2008): Auf der Suche nach neuer Sicherheit. VS Verlag. S. 265.

74 Singelstein, Tobias/Stolle, Peer (2012): Die Sicherheitsgesellschaft. Soziale Kontrolle im 21. Jahrhundert, 3. Auflage, Wiesbaden: VS Verlag. S. 171. 
men für diese Studie dient (vgl. Kapitel 2). So untersuchen Fischer und Masala den Wandel der Sicherheitskultur anhand von Versicherheitlichungssdynamiken im zivilen Luftverkehr. ${ }^{75}$ Matthias Schulze beschreibt mithilfe der Theorie der Versicherheitlichung die Konstruktion von Bedrohung im sicherheitspolitischen Diskurs in Deutschland. ${ }^{76}$

\subsubsection{Privatisierung von Sicherheit}

Eine weitere Folge des Wandels in der Sicherheitskultur ist eine zunehmende Privatisierung von Sicherheit. In Deutschland sind mehr Menschen im privaten Sicherheitsgewerbe beschäftigt als bei der Polizei. ${ }^{77}$ Die Ursachen sind vielfältig: Einerseits führt eine »Differenzierung von Sicherheitsleistungen« sowie die oben bereits diskutierte präventive Wende in der Polizeiarbeit da$\mathrm{zu}$, dass eine stärkere fachliche Spezialisierung notwendig geworden ist. Diese neuen Bedürfnisse werden teilweise an private Dienstleister ausgelagert. ${ }^{78}$ Andererseits sind private Sicherheitsdienste kostengünstiger, ${ }^{79}$ so werden sie mitunter vom Staat für Großveranstaltungen engagiert. ${ }^{80}$ Und schließlich ist durch das Entstehen von quasi privatisierten öffentlichen Räumen (Bahnhöfe, Shoppingmalls) eine neue Nachfrage nach privaten Sicherheitsdiensten entstanden. ${ }^{81}$

75 Vgl. Fischer, S./Masala, C. (2011): Wandelt sich so Sicherheitskultur? Versicherheitlichungsdynamiken und Sicherheitsmaßnahmen am Beispiel des zivilen Luftverkehrs. Sicherheit und Frieden (S F)/Security and Peace, 29(2). S. 109-116.

76 Vgl. Schulze, Matthias (2012): Die Sprache der (Un-)Sicherheit. Die Konstruktion von Bedrohung im sicherheitspolitischen Diskurs der Bundesrepublik Deutschland. Band 51, Marburg: Tectum Verlag. Vgl. hier außerdem: Stöhr, F. (2013): Bedrohungswahrnehmung, Gefahrenabwehr und der Wandel der strategischen Kultur: Wie Politik und Cesellschaft in Deutschland auf den islamistischen Terrorismus reagieren. Jahrbuch Terrorismus, 6. S. 311-324.

77 Vgl. Kreissl, Reinhard (2010): Privatisierung von Sicherheit. In: Zoche/Kaufmann/Haverkamp (Hg.) (2010): Zivile Sicherheit. Cesellschaftliche Dimensionen gegenwärtiger Sicherheitspolitiken. Bielefeld: transcript. S. 267.

78 Vgl. ebd., S. 270.

79 Vgl. ebd., S. 273.

80 Vgl. Feltes, Thomas (2008): Akteure der Inneren Sicherheit: Vom Öffentlichen zum Privaten. In: Lange/Ohly/Reichertz (Hg.) (2008): Auf der Suche nach neuer Sicherheit. Wiesbaden: VS Verlag. S. 106.

81 Vgl. Kreissl, Reinhard (2010): Privatisierung von Sicherheit. In: Zoche/Kaufmann/Haverkamp (Hg.) (2010): Zivile Sicherheit. Gesellschaftliche Dimensionen gegenwärtiger Sicherheitspolitiken. Bielefeld: transcript. S. 270. 
Die zunehmende Privatisierung von Sicherheit wird von den Autor*innen kritisch diskutiert. Daase und Deitelhoff beschreiben eine Veränderung der Anreizstruktur zur Herstellung von Sicherheit, wenn Sicherheit zur Ware wird. Eine in der Bevölkerung wahrgenommene Unsicherheit sichere den Unternehmen der Sicherheitsbranche Gewinne. ${ }^{82}$ Schneiker und Joachim weisen zudem auf die demokratietheoretischen Folgen der Sicherheitsprivatisierung hin. So erfolge die Gewährleistung von Sicherheit hier nicht demokratisch, sondern nur für diejenigen, die dafür bezahlen können. ${ }^{83}$ Sicherheit als Produkt widerspreche dem Grundgedanken des Rechtsstaats, der durch das Monopol auf legitime physische Gewaltanwendung bestehe. ${ }^{84}$ Wenn Sicherheit von einem öffentlichen $\mathrm{zu}$ einem privaten Gut wird, so gehe die NichtAusschließbarkeit und die Nicht-Rivalität von Sicherheit verloren. ${ }^{85}$

Schon aufgrund des Begriffs denken wir bei einer Privatisierung von Sicherheit an private Unternehmen, die eine ehemals staatseigene Aufgabe übernehmen. Aber Privatisierung kann auch in einem ganz wörtlichen Sinne bedeuten, dass die Verantwortung für Sicherheit in die Hände von Einzelpersonen gelegt wird. So beschreibt Reinhard Kreissl, analog zum Rückzug des Staates aus der Verantwortung für soziale Sicherheit, den Rückzug des Staates aus der Gewährleistung von privater Sicherheit, beispielsweise bezüglich des Schutzes vor Einbrechern. Vom verantwortungsvollen Individuum werde erwartet, selbst für seine Sicherheit zu sorgen. Kreissl verweist in diesem Zusammenhang auf Nachbarschaftswachen. ${ }^{86}$

Die Gründungen von Bürgerwehren lassen sich also als Privatisierung von Sicherheit beschreiben. In einer Gesellschaft, in der die Gewährleistung von

82 Vgl. Schneiker, Andrea (2017): Privatisierung von Sicherheit - mehr Sicherheit für alle oder exklusive Sicherheit für wenige? In: Diagonal. Zeitschrift der Universität Siegen. Cöttingen: V\&R. S. 83.

83 Vgl. Schneiker, Andrea/Joachim, Jutta (2018): Privatisierung von Sicherheit in Deutschland- Einleitung in den Themenschwerpunkt. In: ZeFKo, Jahrgang 7 (2018), Heft 1. S. 45 .

84 Vgl. Priester, Karin (2010): Innere Sicherheit und Bürgerwehren als Politikum. In: Neue Cesellschaft, Frankfurter Hefte, Nr. 12/2010. S. 33f.

85 Vgl. Daase, Christopher/Deitelhoff, Nicole (2013): Privatisierung der Sicherheit. Eine sozialwissenschaftliche Expertise. In: Schiller/Gerhold/Steiger/Jäckel (Hg.) (2013): Forschungsforum Öffentliche Sicherheit. Schriftenreihe Sicherheit Nr. 11, September 2013. S. 25.

86 Vgl. Kreissl, Reinhard (2010): Privatisierung von Sicherheit. In: Zoche/Kaufmann/Haverkamp (Hg.) (2010): Zivile Sicherheit. Cesellschaftliche Dimensionen gegenwärtiger Sicherheitspolitiken. Bielefeld: transcript. S. $274 \mathrm{f}$. 
Sicherheit ausgelagert wird, erscheint die Selbstorganisation von Bürger*innen als Reaktion auf unklare Zuständigkeitsverhältnisse. ${ }^{87}$

\subsubsection{Bürgerwehren als Community Policing}

Neben Privatisierungstendenzen ist die partizipative Polizeiarbeit eine weitere Reaktion auf neue Unsicherheitswahrnehmungen und den Wandel der Sicherheitskultur. So übernehmen in Krisenzeiten Bürgerinnen und Bürger mitunter staatliche Aufgaben, beispielsweise bei Naturkatastrophen. Soziale Medien erleichtern hier die Mobilisierung und Koordinierung der Freiwilligen. ${ }^{88}$ Aber auch in der Herstellung alltäglicher Innerer Sicherheit werden zunehmend Bürger*innen eingebunden. In den USA beispielsweise ist unter dem Begriff Community Policing ${ }^{89}$ die Zusammenarbeit mit lokalen Nachbarschaftswachen verbreitet. ${ }^{90}$ Community Policing beschreibt eine Strategie der Kriminalitätsprävention auf lokaler Ebene, die auf eine »Aktivierung« von Bürger*innen setzt. ${ }^{11}$ Van Ooyen beschreibt für den deutschen Kontext Bürgerwehren als Form von Community Policing. ${ }^{92}$

Diesem Ansatz liegt ein ausgedehnter Polizeibegriff zugrunde, der auf eine gegenseitige soziale Kontrolle von Bürger*innen setzt. Das Konzept beinhaltet, dass der Staat und seine Institutionen hier die Kontrolle haben. Sie bestimmen die Art und Weise der Einbindung von Bürger*innen und setzen Grenzen. Dementsprechend beschreibt van Ooyen nur Bürgerwehren, die unter staatlicher Kontrolle stehen oder direkt von staatlichen Organen initiiert wurden: Hilfspolizist*innen sowie lokale Sicherheitspartnerschaften und die

87 Vgl. Priester, Karin (2010): Innere Sicherheit und Bürgerwehren als Politikum. In: Neue Gesellschaft, Frankfurter Hefte, Nr. 12/2010. S. 35.

88 Vgl. Liegl, Michael/Büscher, Monika (2016): Resiliente Gesellschaft? Bürgerpartizipation, Digital Volunteers und (g)lokale Selbsthilfe im Katastrophenschutz. In: Zoche, Peter et.al. (2016): Grenzenlose Sicherheit? Gesellschaftliche Dimensionen der Sicherheitsforschung. Berlin: LIT Verlag. S. 253-270.

89 Vgl. zu den Möglichkeiten einer Übertragung des Konzepts aus dem USamerikanischen Kontext auf Deutschland: Jahn, Thomas (2003): Bürger- und gemeinwesenorientierte Polizeiarbeit. Hamburg: Kovac.

90 Vgl. Wurtzbacher, Jens (2008): Urbane Sicherheit und Partizipation. Stellenwert und Funktion bürgerschaftlicher Beteiligung an kommunaler Kriminalprävention. Opladen: Leske + Budrich. S. $242 f$.

91 Vgl. Ooyen, Robert Christian van (2007): Öffentliche Sicherheit und Freiheit: Studien zu Staat, Polizei und wehrhafter Demokratie. Baden-Baden: Nomos. S. 120.

92 Vgl. ebd., S. $122 \mathrm{ff}$. 
Sicherheitswacht in Bayern. Hubert Beste beschreibt außerdem lokale Präventionsräte, die im Sinne einer aufsuchenden Sozialarbeit Probleme ohne Einschalten der Polizei lösen sollen. ${ }^{93}$ Beide Autoren beschreiben die Bürgereinbindung kritisch, als »symbolische Politik, die fürsorgliche Betriebsamkeit und politische Verantwortlichkeit vortäuscht. " ${ }^{94}$ Van Oyen erkennt zwar eine Zunahme des subjektiven Sicherheitsgefühls durch Community Policing, warnt jedoch bei der Einbindung von Bürger*innen in die Polizeiarbeit vor einem »Freund-Feind «-Denken, das den »Anderen« als »Störer « definiert. ${ }^{95}$ Auch Braun bewertet die Einbindung von Laien in die Polizeiarbeit sehr kritisch. Anstatt durch »Billigpolizisten« bei der staatlichen Polizei Kräfte freiwerden zu lassen, sei eine ungleiche Verteilung von Sicherheit zu befürchten. Außerdem bestehe die Gefahr, dass die Bürger*innen ihre Befugnisse überschritten. ${ }^{96}$

Ronald Hitzler beschrieb bereits in den 1990er Jahren die Einbindung von Bürger*innen als Hilfspolizisten, um neuen Unsicherheitsgefühlen in der Bevölkerung zu begegnen. Hitzler zufolge ist diese Bürgerbeteiligung an der Herstellung Innerer Sicherheit jedoch kontraproduktiv:

»Die unter Polizeiaufsicht agierenden Bürgerwachten repräsentieren im wesentlichen also wohl den politischen Versuch, das (potentiell anarchistische) Phänomen präventivrepressiver Selbsthilfe in einer Art >Mitverantwortungく des Bürgers für die Ordnungs- und Kontrollinteressen des Staates zu disziplinieren. ${ }^{97}$

93 Vgl. Beste, Hubert (2000): Morphologie der Macht. Urbane »Sicherheit« und die Profitorientierung sozialer Kontrolle. Opladen: Lecke + Budrich. S. $311 \mathrm{ff}$.

94 Beste, Hubert: Bürgeraktivierung im System »innerer Sicherheit«. Die Wiederentdeckung des Lokalen. In: Bürgerrechte \& Polizei/CILIP 66 (2/2000). S. 8.

95 Vgl. Ooyen, Robert Christian van (2007): Öffentliche Sicherheit und Freiheit: Studien zu Staat, Polizei und wehrhafter Demokratie. Baden-Baden: Nomos. S. 124ff.

96 Vgl. Braun, Frank (2014): Wie bürgerliche Freiheitsrechte durch eine Kommunalisierung und »Laisierung « von Polizeiarbeit gefährdet werden. In: Abt, Jan et al. (Hg.) (2014): Dynamische Arrangements städtischer Sicherheit. Akteure, Kulturen, Bilder. Wiesbaden: VS Verlag. S. $173 \mathrm{f}$.

97 Hitzler, Ronald (1994): Die neuen Vigilanten. Über Formen der Bewältigung alltäglicher Verunsicherung, in: Bürgerrechte \& Polizei 48: 2. S. 7of. 
Hitzler analysiert die in den frühen 1990er Jahren auftretenden Bürgerwehren als neue soziale Sicherheitsbewegung ${ }^{98}$ und die in einigen Bundesländern entstehenden Sicherheitspartnerschaften bzw. Sicherheitswachten als Reaktion des Staates auf dieses Phänomen. So versuchten Polizei und Staat durch eine Einbindung von Bürger*innen in polizeiliche Sicherheitskonzepte das subjektive Sicherheitsgefühl in der Gesellschaft zu verbessern. Insbesondere, was die Gewährleitung von Sicherheit und Ordnung im öffentlichen Raum angeht, bildeten polizeilich organisierte Bürgerstreifen eine kostengünstige Alternative zu einem Ausbau polizeilicher Präsenz.${ }^{99}$ Wurtzbacher hingegen plädiert dafür, Staat und Bürger in Bezug auf das Politikfeld Sicherheit nicht als getrennte Seiten wahrzunehmen. Vielmehr werde Sicherheit als soziale Sphäre gesellschaftlich hergestellt. Bürgerschaftliches Engagement für Sicherheit ist nach Wurtzbacher Ausdruck der sozialen Gestaltbarkeit von Sicherheit und auf lokaler Ebene daher eine demokratische Partizipationsmöglichkeit. ${ }^{100}$

Im Forschungsfeld ziviler Sicherheit werden Bürgerwehren als Form des Community Policing eingeordnet. Das greift jedoch nur für Bürgerwehren, die mit dem Staat zusammenarbeiten. Insbesondere Bürgerwehren, die sich in den letzten Jahren gründeten, kommen aufgrund ihrer politischen Ausrichtung mitunter nicht für den Staat als Sicherheitspartner infrage. Diese Bürgerwehren werden im Kontext des Vigilantismus analysiert. Staatstheoretisch formuliert, stellen sie das Monopol der legitimen Gewaltausübung des Staates infrage.

\subsubsection{Eine staatstheoretische Einordnung des Phänomens Bürgerwehren}

In der Legitimation des modernen Nationalstaats diente Sicherheit den Theoretikern der Neuzeit als zentrales Motiv für die Bürger, staatliche Herrschaft zu akzeptieren. ${ }^{101}$ Die Fähigkeit des Staates, Sicherheit zu gewährleisten,

98 Vgl. Hitzler, Ronald (1993): Bürger machen mobil. Über die neue soziale SicherheitsBewegung, in: Forschungsjournal Neue Soziale Bewegungen 3-4. S. 16-27.

99 Vgl. Ooyen, Robert Christian van (2007): Öffentliche Sicherheit und Freiheit. Studien zu Staat, Polizei und wehrhafter Demokratie. Baden-Baden: Nomos. S. 123.

101 Vgl. bspw. Hobbes, Thomas (1651): Leviathan. Hamburg (1996): Felix Meiner Verlag. 
ist das zentrale Merkmal, mit dem sich der moderne Nationalstaat gegenüber anderen politischen Organisationsformen durchsetzte. ${ }^{102}$ Sicherheit zu schaffen ist somit eine zentrale Größe, an der die Legitimation staatlicher Herrschaft gemessen wird. Überlegungen, die Legitimität als zentrale Ressource für moderne Nationalstaaten beschreiben, gehen auf Max Weber zurück. Nach Weber sucht jede Herrschaft, »den Glauben an ihre >Legitimitä‘ $\mathrm{zu}$ wecken und zu pflegen ${ }^{103}{ }^{103}$ Dieser »Legitimitätsglaube « innerhalb der Gesellschaft ist das Fundament der Ordnung im modernen Nationalstaat.

Im modernen Nationalstaat hat der staatliche Verwaltungsapparat das Monopol auf die Ausübung des legitimen physischen Zwangs. Dieses staatliche Gewaltmonopol setzt voraus, dass der Herrschaftsapparat des Staates jegliche Ausübung der gewalttätigen Selbsthilfe zerschlägt. ${ }^{104}$ Dabei stellt privat ausgeübte Gewalt erst einmal nicht das staatliche Gewaltmonopol infrage. Wird sie zur Anzeige gebracht, wird die Gewaltausübung gemäß geltenden Rechts bestraft und bestätigt somit das staatliche Gewaltmonopol, anstatt es infrage zu stellen. ${ }^{105}$ Bürgerwehren hingegen proklamieren, das Recht durchsetzen zu wollen, weil der Staat in ihren Augen Lücken aufweist in der Aufrechterhaltung seiner Funktion als singulärer Sicherheitsgarant.

Dennoch unterscheidet sich die Macht, die Bürgerwehren ausüben, von staatlicher Macht. Heinrich Popitz versteht unter Herrschaft institutionalisierte Macht. Im Prozess der Institutionalisierung von Macht vollzieht sich eine Entpersonalisierung von Macht. Die Macht hängt nicht mehr an der Person, die sie ausübt, sondern wird stattdessen an eine soziale Position oder

102 Vgl. Weber, Max (1922): Wirtschaft und Cesellschaft. Grundriß der verstehenden Soziologie, J.C.B. Mohr (Paul Siebeck). 5. Aufl. 1980 [1922], Tübingen. S. 29f. Zitiert nach: Herschinger/Jachtenfuchs/Kraft-Kasack (2010): Das staatliche Cewaltmonopol: Internationalisierung ohne Politisierung. In: Zoche/Kaufmann/Haverkamp (Hg.) (2010): Zivile Sicherheit. Cesellschaftliche Dimensionen gegenwärtiger Sicherheitspolitiken. Bielefeld: transcript. S. 227-246.

103 Weber, Max (1922): Wirtschaft und Cesellschaft. 5. revidierte Auflage, besorgt von Johannes Winkelmann (1985). Mohr (Paul Siebeck). Tübingen: Verlag Paul Siebeck. S. 122.

104 Vgl. von Throtha, Trutz (1995): Ordnungsformen der Cewalt oder Ausrichtung auf das Ende des staatlichen Cewaltmonopols. In: Nedelmann, Brigitta (1995): Politische Institutionen im Wandel. Opladen: Westdeutscher Verlag. S. 133.

105 Vgl. Crimm, Dieter (2002): Das staatliche Cewaltmonopol. In: Heitmeyer, Wilhelm/Hagan, John (Hg.) (2002): Internationales Handbuch der Gewaltforschung. Wiesbaden: Westdeutscher Verlag. S. $1303 \mathrm{f}$. 
Funktion gekoppelt. Das unterscheidet staatliche Herrschaft von der Machtausübung durch vom Staat unabhängig agierende Akteure. ${ }^{106}$ Bürgerwehren reißen Macht an sich, ohne sie in legitimer Weise bekommen zu haben. Sie üben Macht aus, um Recht zu setzen. ${ }^{107}$ Der demokratische Rechtsstaat mit funktionierender Gewaltenteilung hingegen übt Macht aus, um Recht aufrechtzuerhalten.

Das ist jedoch eine idealtypische Beschreibung staatlicher Herrschaft. Gegenwärtig erleben wir eine Verschiebung von zwischenstaatlichen Konflikten hin zu einer »Globalisierung des Politischen« mit transnationalen Konfliktlinien. Das Ideal des Staats als ausübende Institution politischer Herrschaft erodiert. ${ }^{108}$ Hier zeigt sich, dass die Legitimität staatlicher Ordnung kein ein für alle Mal feststehender Zustand ist, sondern ein andauernder Prozess der Legitimitätsaufrechterhaltung. Bürgerwehren veranschaulichen diese Fragilität moderner sozialer Ordnungen. ${ }^{109}$

Im staatstheoretischen Diskurs werden Bürgerwehren als Phänomen des Vigilantismus betrachtet. Insbesondere dann, wenn Bürgerwehren (politisch motiviert) Gewalt ausüben, werden sie zudem als terroristische Gruppen und rechtsextreme Organisationsform begriffen. Um den staatstheoretischen Diskurs über Bürgerwehren abzubilden, wird daher nun zunächst der Forschungsstand zum Vigilantismus dargelegt. Anschließend werden Bürgerwehren als rechtsextreme Organisationsform zur strategischen Machtgewinnung durch Präsenz und Kontrolle im öffentlichen Raum diskutiert.

\subsubsection{Bürgerwehren als Phänomen des Vigilantismus}

Die wenigen Studien, die es in Deutschland über zeitgenössische Bürgerwehren - die auch jenseits staatlicher Kontrolle agieren - gibt, analysieren das

106 Vgl. Popitz, Heinrich (1992): Phänomene der Macht, 2., stark erw. Aufl., Tübingen: Mohr. S. $233 \mathrm{ff}$.

107 Vgl. Schmidt-Lux, Thomas (2018): Anspruch und Glauben. Vigilantismus als Herausforderung staatlicher Legitimität. In: Zabel, Benno (2018): Philosophie der Republik. Tübingen: Verlag Paul Siebeck. S. 142.

108 Vgl. Bonacker, Thorsten (2007): Der Kampf der Interpretationen - Zur Konflikthaftigkeit der politischen Moderne. In: Ders./Reckwitz, Andreas (Hg.): Kulturen der Moderne. Frankfurt/New York: Campus Verlag. S. 214f.

109 Vgl. Schmidt-Lux, Thomas (2018): Bürgerwehren als kollektive Akteure im Feld von Sicherheit und Recht. In: ZeFKo 7 Jg. (2018), Heft 1. S. 131. 
Phänomen als Vigilantismus. ${ }^{110}$ Insbesondere sind hier die Studien von Thomas Schmidt-Lux und Matthias Quent zu nennen. ${ }^{111}$ Des Weiteren hat Ronald Hitzler wie oben erwähnt die Einbindung von Bürgern in lokale Polizeiarbeit in den 1990er Jahren untersucht. ${ }^{112}$

110 Der Begriff »Vigilantismus« ist eine direkte Übersetzung des englischen Begriffs »Vigilantism«, der im englischen Sprachgebrauch verbreitet ist. »Vigilantism« hat in den USA geschichtlich eine größere Bedeutung. Weil die koloniale Einnahme des Landes schneller fortschritt als der Aufbau eines Polizeiapparates, entstanden in Crenzgebieten Organisationen, die für sich in Anspruch nahmen, Recht durchzusetzen und abweichendes Verhalten zu bestrafen. Vgl. Kirsch, Andreas (2000): Cewalt bei sportlichen Großveranstaltungen. Frankfurt: Peter Lang. S. 21.

Im Deutschen hingegen ist der Begriff »Vigilantismus« nicht gebräuchlich. Die Forschungsdebatte über Bürgerwehren in Deutschland folgt der US-amerikanischen Einordnung des Phänomens als »Vigilantism« und verwendet daher den Begriff Vigilantismus. In der deutschen Forschungsdebatte werden neben Bürgerwehren jedoch auch weitere Phänomene unter der Kategorie Vigilantismus diskutiert. Gräfe beschreibt beispielsweise den Rechtsterrorismus der Bundesrepublik als Vigilantismus. Vgl. Gräfe, Sebastian (2017): Fünf Jahrzehnte Rechtsterrorismus in der Bundesrepublik Deutschland. Von der »Europäischen Befreiungsfront« bis zum »Nationalsozialistischen Untergrund«. Jahrbuch Terrorismus, 8. S. 234.

Vgl. Quent, Matthias (2016): Selbstjustiz im Namen des Volkes: Vigilantischer Terrorismus. In: APuZ 24-25/2016. S. 20-26. Quent, Matthias (2016): Bürgerwehren. Hilfssheriffs oder inszenierte Provokation? Amadeus Antonio Stiftung. Cottbus: Druckzone. Schmidt-Lux, Thomas (2012): Vigilantismus. Ein Phänomen der Grenze? In: Kriminologisches Journal, Heft 2, 2012. S. 118- 132. Schmidt-Lux, Thomas (2013): Vigilantismus als politische Gewalt. Eine Typologie. In: behemoth 6, Heft 1, 2013. S. 98-117. SchmidtLux, Thomas (2013): Jenseits von Batman. Schlüsselfiguren des Vigilantismus. In: Forschungsjournal Soziale Bewegungen, Heft 4, 2013. S. S. 64-71. Schmidt-Lux, Thomas (2018): Bürgerwehren als kollektive Akteure im Feld von Sicherheit und Recht. In: ZeFKo 7, 2018, 1. S. 131-163. Schmidt-Lux, Thomas (2018): Anspruch und Clauben. Vigilantismus als Herausforderung staatlicher Legitimität. In: Zabel, Benno (2018): Philosophie der Republik. Siebeck. Schmidt-Lux, Thomas (2017): Cerechte Strafe. Legitimationskonflikte um vigilante Cewalt. Weinheim: Beltz.

112 Vgl. Hitzler, Ronald (1993): Bürger machen mobil. Über die neue soziale SicherheitsBewegung, in: Forschungsjournal Neue Soziale Bewegungen 3-4, S. 16-27.

Hitzler, Ronald (1994): Die neuen Vigilanten. Über Formen der Bewältigung alltäglicher Verunsicherung. In: Bürgerrechte \& Polizei 48: 2. S. 67-71. Hitzler, Ronald (1996): Der in die Polizeiarbeit eingebundene Bürger. Zur symbolischen Politik mit der bayrischen Sicherheitswacht. In: Reichertz, Jo/Schröer, Norbert (Hg.): Qualitäten polizeilichen Handelns. Beiträge zu einer verstehenden Polizeiforschung. Opladen: Westdeutscher Verlag. S. 30-47. 
Insgesamt gibt es wenig Forschung über das Phänomen und kaum theoretische Ansätze. Der erste Ansatz, der das Phänomen in den USA theoretisch $\mathrm{zu}$ fassen suchte, ist die >Frontier - Theorie von Brown. ${ }^{113}$ Sie bezieht sich insbesondere auf die Zeit des Amerikanischen Bürgerkriegs und beschreibt Vigilantismus als ein Phänomen, das in Grenzregionen mit wenig staatlicher Kontrollmacht entsteht. Die Theorie erweist sich für die Erklärung spontaner Zusammenrottungen wie Lynchmobs als fruchtbar, kann jedoch nur begrenzt auf andere Regionen übertragen werden.

Eine andere Definition liefert Kowalewski. Er fasst unter Vigilantismus "Aktivitäten zur Unterdrückung von abweichendem Verhalten (Devianz) anderer Bürger seitens Privatpersonen «. ${ }^{114}$ Dabei kann das als abweichend wahrgenommene Verhalten drei Formen annehmen: kriminelle Devianz, kulturelle Devianz und politische Devianz. Sowohl die Vigilanten als auch die Devianten können individuell oder als Kollektive auftreten. ${ }^{115}$

Die »Theorie der Gegenbewegung« von Kowalewski erklärt Vigilantismus mit einer Gegenbewegung ${ }^{116}$ in Bezug auf gesellschaftliche Veränderungen, die als Bedrohung für die eigene Lebensform empfunden werden und mittels vigilanter Aktivitäten zurückgedrängt werden sollen. ${ }^{117}$ Die An- oder Abwesenheit staatlicher Kontrolle in der Region, in der die Vigilanten agieren, hat in Kowalewskis Theorie keinen Einfluss auf das Verhalten der Vigilanten. Kowalewski bewertet Vigilantismus also nicht als Gegenbewegung zum Staat - im Gegenteil: Staatliche Akteure tolerieren oder unterstützen vigilante Gruppen mitunter, weil sie ein kostengünstiges Mittel zur Kontrolle von Devianten darstellen. ${ }^{118}$ Das unterscheidet Kowalewski von Brown, der expli-

113 Vgl. hierzu Brown, Richard (1975): Strain of Violence. Historical Studies of American Violence and Vigilantism. New York: Oxford University Press.

Sowie: McGrath, Roger (1984): Gunfighters, Highwaymen and Vigilantes. Berkley: University of California Press.

114 Kowalewski, David (2002): Vigilantismus. In: Heitmeyer/Hagan (Hg.): Internationales Handbuch der Gewaltforschung. VS Verlag. S. 426.

115 Vgl. ebd., S. 426.

116 Hier schließt Lee Johnston an und begreift Vigilanten als soziale Bewegung. Vgl. Johnston, Lee (1996): What is Vigilantism? In: The British Journal of Criminology. Jg. 36, Nr. 2 (1996). S. 220-236.

117 Vgl. Kowalewski, David (2002): Vigilantismus. In: Heitmeyer/Hagan (Hg.): Internationales Handbuch der Cewaltforschung. VS Verlag. S. 429.

118 Vgl. ebd., S. 429. 
zit die Abwesenheit staatlicher Kontrollmacht als Voraussetzung für Vigilantismus beschrieb.

Obwohl die >Frontier-Theorie in der Forschungsdebatte als überholt gilt, greift Schmidt-Lux die Theorie 2012 in seiner Studie über eine Bürgerwehr im deutsch-tschechischen Grenzgebiet wieder auf. ${ }^{119}$ Die Grenzlage führt nach Schmidt-Lux dazu, dass sich Bürger*innen dort eher als abgehängt wahrnehmen würden. Er plädiert dafür, die >Frontier<-Theorie zu modifizieren, sodass mit »Grenzregion« nicht mehr die geografische Nähe einer Region zu den Staatsgrenzen gemeint ist, ${ }^{120}$ sondern eine von den Vigilanten selbst wahrgenommene Ferne von staatlichen Instanzen. ${ }^{121}$ Thomas Schmidt-Lux analysiert aber auch die staatliche Seite des Phänomens und untersucht die Legitimationskonflikte, in die staatliche Ordnungen geraten, wenn Bürgerwehren auftreten. $^{122}$

\subsubsection{Bürgerwehren als rechtsextreme Organisationsform}

Matthias Quent beschreibt Bürgerwehren, wenn sie Gewalt anwenden, als Form von Terrorismus ${ }^{123}$ und beruft sich dabei auf die Terrorismus-Studien von Peter Waldmann. Waldmann beschreibt Terrorismus als Kommunikationsstrategie:

»Dem Terroristen geht es nicht um den eigentlichen Zerstörungseffekt seiner Aktionen. Diese sind nur ein Mittel, eine Art Signal, um einer Vielzahl

119 Vgl. Schmidt-Lux, Thomas (2012): Vigilantismus. Ein Phänomen der Grenze? In: Kriminologisches Journal, Heft 2, 2012. S. 118-132.

120 Vgl. ebd. S. 130.

121 Schmidt-Lux beschreibt zudem die Rolle von Schlüsselfiguren in vigilanten Gruppen. Die Rolle eines »zivilisierenden Anführers « ist demnach wichtig, um zwischen Staat, Cesellschaft und Bürgerwehr zu vermitteln. Vgl. ebd., S. 127.

Sowie: Schmidt-Lux, Thomas (2013): Jenseits von Batman. Schlüsselfiguren des Vigilantismus. In: Forschungsjournal Soziale Bewegungen, Heft 4, 2013. S. 79.

122 Vgl. Schmidt-Lux, Thomas (2018): Anspruch und Clauben. Vigilantismus als Herausforderung staatlicher Legitimität. In: Zabel, Benno (2018): Philosophie der Republik. Siebeck. S. 142-155.

123 Quent merkt jedoch an, dass die meisten Bürgerwehren sich als Protestgruppen formieren. Sie drohen an, das Recht in die eigenen Hände zu nehmen, entwickeln jedoch keine gewalttätigen Aktivitäten. Vgl. Quent, Matthias (2016): Selbstjustiz im Namen des Volkes: Vigilantischer Terrorismus. In: Aus Politik und Zeitgeschichte: Terrorismus, APuZ 24-25/2016. S. 26. 
von Menschen etwas mitzuteilen. Terrorismus, das gilt es festzuhalten, ist primär eine Kommunikationsstrategie. $\ll^{124}$

Wie Terroristen richten sich Bürgerwehren meist gegen bestimmte gesellschaftliche Gruppen, nicht so sehr gegen den Staat an sich. Insbesondere die Bürgerwehren, die sich als Reaktion auf die Flüchtlingsbewegungen 2015/16 gründeten, erteilen dem modernen Nationalstaat keine generelle Absage; vielmehr formulierten sie ein Misstrauen in dessen Durchsetzungskraft. Ihr Handeln verteidigt die "alte Ordnung«, auch wenn das staatliche Gewaltmonopol dabei vorübergehend infrage gestellt werden muss. ${ }^{125}$

Bürgerwehren sind nicht gegen die Mehrheitsgesellschaft gerichtet, sie sind Teil der Zivilgesellschaft und agieren aus ihr heraus. Sie versuchen sich als Sicherheitsakteure zu etablieren und präsentieren sich als engagierte Bürger, ${ }^{126}$ die einen Beitrag für die Gemeinschaft leisten. Aber wie Roland Roth in seinem Aufsatz »Die dunklen Seiten der Zivilgesellschaft « ${ }^{127}$ zeigt, fördert ein zivilgesellschaftliches Engagement an sich nicht die demokratische Kultur. So lässt sich beispielsweise in den neuen Bundesländern zeigen, dass nach der Wende zwar die Anzahl der Vereine und der engagierten Menschen stieg, das Vertrauen in demokratische Institutionen jedoch gleichzeitig abnahm. ${ }^{128}$

Vigilantismus richtet sich also gegen einzelne gesellschaftliche Gruppen. Bei der Gewaltausübung seitens vigilanter Gruppen, die mit Machtressourcen ausgestattet sind, gegenüber einem anderen Kollektiv oder Einzelpersonen werden dessen Mitglieder meist stereotyp als mit bestimmten Eigenschaften

124 Vgl. Waldmann, Peter (2005): Terrorismus. Provokation der Macht, 2. vollst. überarbeitete Auflage, Hamburg: Murmann. S. 15.

125 Vgl. Quent, Matthias (2016): Selbstjustiz im Namen des Volkes: Vigilantischer Terrorismus. In: Aus Politik und Zeitgeschichte: Terrorismus, APuZ 24-25/2016. S. 20.

126 Vgl. hierzu auch: Hoffmann, Anika (2019): Bürgerwehren und ihre Bedeutung im öffentlichen Raum. Abweichendes Verhalten als kriminologisch relevantes Phänomen. Wiesbaden: VS Verlag. S. $137 \mathrm{ff}$.

127 Vgl. Roth, Roland (2003): Die dunklen Seiten der Zivilgesellschaft. Grenzen einer zivilgesellschaftlichen Fundierung von Demokratie. In: Forschungsjournal NSB 16-2003-2. S. 59-73.

128 Vgl. Roth, Roland (2003): Die dunklen Seiten der Zivilgesellschaft. Grenzen einer zivilgesellschaftlichen Fundierung von Demokratie. In: Forschungsjournal NSB 16-2003-2. S. 63. 
ausgestattet betrachtet. ${ }^{129}$ Den Staat greifen vigilante Gruppen nur deshalb an, weil er vermeintlich in die falschen Hände gefallen ist. ${ }^{130}$ Ein Beispiel für einen solchen Angriff ist der Mord am hessischen CDU-Politiker Walter Lübcke. $^{131}$

In der Legitimationslogik vigilanter Gewalt, die sich gegen bestimmte Gruppen richtet, ist eine Verquickung von Kriminalitätsbekämpfung und Rassismus $\mathrm{zu}$ beobachten. So diente beispielsweise der »rape myth«, der Vergewaltigungsmythos, als Legitimation für die Lynchmorde von Weißen an Schwarzen US-Amerikanern. Im Süden US-Amerikas zu Zeiten des Bürgerkriegs argumentierte die Weiße Mehrheitsgesellschaft, der Staat sei zu schwach, die Gesetze durchzusetzen, sodass sie kriminelles Verhalten (der Schwarzen) selber bestrafen müssten. ${ }^{132}$ Hier zeigt sich, dass auch die Kategorie »Geschlecht « in Untersuchungen vigilanter Gruppen mitunter einen relevanten Einflussfaktor darstellt.(vgl. nächstes Unterkapitel 1.2.2.3).

Insbesondere Matthias Quent verweist auf den Zusammenhang von rechtsextremem Denken und Bürgerwehren. Ihm zufolge kann eine wahrgenommene Illegitimität staatlicher Gewaltausübung zu einer Radikalisierung von Bürgerinnen und Bürgern führen, die auch in Gewaltausbrüchen enden kann. ${ }^{133}$ Die Analyse von Matthias Quent zeigt, dass nicht wenige Bürgerwehren ein rechtsextremes Weltbild vertreten und mitunter direkt zu Gewalt aufrufen. ${ }^{134}$

129 Vgl. Schwalb, Benjamin/Paul, Axel T. (2015): Nicht-organisierte kollektive Cewalt. In: Cewaltmassen. Über Eigendynamik und Selbstorganisation kollektiver Cewalt. Hamburg: Hamburger Edition. S. 395.

130 Vgl. Quent, Matthias (2016): Selbstjustiz im Namen des Volkes: Vigilantischer Terrorismus. In: Aus Politik und Zeitgeschichte: Terrorismus, APuZ 24-25/2016. S. 23.

131 Dem Mord an Lübcke ging eine Hetze im Netz voraus. Der rechtsextreme Blog »Politically Incorrect« veröffentlichte seine Privatadresse. Der Journalist Robert Andreasch veröffentlichte Screenshots des Blogs www.pi-news.net, auf denen die Veröffentlichung der Adresse zu sehen ist: Vgl. https://twitter.com/robertandreasch/status/11355 84027556155392 (Zugriff am 2.1.2021).

132 Zur Lynchjustiz in den USA vgl. Berg, Manfred (2011): Popular justice: a history of lynching in America. Chicago: Dee. S. $98 \mathrm{f}$.

133 Vgl. Schwalb, Benjamin/Paul, Axel T. (2015): Nicht-organisierte kollektive Gewalt. In: Gewaltmassen. Über Eigendynamik und Selbstorganisation kollektiver Gewalt. Hamburg: Hamburger Edition. S. 396.

134 Vgl. Quent, Matthias (2016): Bürgerwehren. Hilfssheriffs oder inszenierte Provokation? Studie für die Amadeus Antonio Stiftung. Cottbus: Druckzone. S. 20-30. 
Wolfgang Keller untersucht in seiner Vergleichsstudie den Einfluss rassistischer Denkweisen auf die Wahrnehmung von Unsicherheiten und findet eine starke Korrelation zwischen rassistischen Einstellungen und dem Problematisieren von Kriminalität. ${ }^{135}$ Diese Tendenz, rassistische Einstellungen auf Kriminalitätsfurcht zu projizieren, sodass Hautfarbe mit Gefahr assoziiert wird, wird insbesondere im US-amerikanischen Kontext von zahlreichen Forscher"innen beschrieben. ${ }^{136}$

Die diskursiven Verschiebungen seit den Migrationsbewegungen nach Deutschland 2015/16 in der öffentlichen Debatte und die breite Akzeptanz neurechter Narrative ist bereits untersucht worden. ${ }^{137}$ Einen Zusammenhang zwischen diesen sprachlichen Verschiebungen und den wachsenden Zahlen rassistisch motivierter Gewalttaten seit 2016 ist jedoch bisher kaum erforscht. Bürgerwehren übernehmen eine Scharnierfunktion: In ihrem Auftreten kristallisiert sich die rassistische Stimmungslage, sie erreicht die praktische Handlungsebene. Auch ohne die Anwendung physischer Gewalt durch Mitglieder der Bürgerwehren ist der Schritt von der sprachlichen auf die Handlungsebene vollzogen.

Die Strategie der extremen Rechten, durch Präsenz im öffentlichen Raum Macht zu erlangen, beschreibt Heitmeyer in seiner Untersuchung »Sozialräumliche Machtversuche des ostdeutschen Rechtsextremismus « ${ }^{138}$

135 Vgl. Keller, Wolfgang (2007): Über den Zusammenhang zwischen fremdenfeindlichen Vorurteilen und kriminalitätsbezogener Unsicherheit. In: Sessar/Stangl/van Swaaningen (Hg.) (2007): Großstadtängste. Untersuchungen zu Unsicherheitsgefühlen und Sicherheitspolitiken in europäischen Kommunen. LIT Verlag. Wien/Berlin: S. 180f.

Vgl. beispielsweise: Chiricos/McEntire/Gertz (2001): Perceived Racial and Ethnic Composition of Neighborhood and Perceived Risk of Crime. In: Social Problems. Vol. 48, No. 3 (August 2001). S. 322-340.

Sowie: Quillian, Lincoln/Pager, Devah (2001): Black Neighbors, Higher Crime? The Role of Racial Stereotypes in Evaluations of Neighborhood Crime. In: American Journal of Sociology Vol. 107, No. 3 (November 2001). S. 717-767.

137 Vgl. bspw. Milzbrand, Björn (2018). Über autoritäre Haltungen in >postfaktischen Zeiten. Opladen/Berlin/Toronto: Verlag Barbara Budrich.

138 Vgl. Heitmeyer, Wilhelm (1999): Sozialräumliche Machtversuche des ostdeutschen Rechtsextremismus. Zum Problem unzureichender politischer Gegenöffentlichkeit in Städten und Kommunen. In: Kalb, Peter/Petry, Christian/Sitte, Karin (Hg.): Rechtsextremistische Jugendliche-was tun? Weinheim: Beltz. S. 47-79.

Siehe auch: Quent, Matthias/Schulz, Peter (2015): Rechtsextremismus in lokalen Kontexten. Vier vergleichende Fallstudien. VS Verlag. S. 22. 
als »Raumgewinne«. ${ }^{139}$ Ziel ist neben der Sichtbarkeit im öffentlichen Raum $^{140}$ insbesondere die Ausweitung von "Angstzonen ${ }^{141}$ Durch eine physische Präsenz, die eine potentielle Gewaltandrohung suggeriert, sollen hier rassifizierte Personen aus dem öffentlichen Raum vertrieben werden.

Wolfgang Sofsky beschreibt Arenen, in denen zeitlich definierte Gewaltprozesse stattfinden, als »Gewalträume ${ }^{142}$ Jörg Baberowski greift den Begriff auf und entwickelt das Konzept ${ }^{143}$ von "Ermöglichungsräumen exzessiver Gewalt «. ${ }^{144}$ Im Gewaltraum wird die körperliche Gewalt zum Sprechakt. ${ }^{145}$ Aber auch ohne die direkte Anwendung physischer Gewalt, wird bei der Erlangung von Kontrolle im öffentlichen Raum die körperliche Präsenz zum Sprechakt. Sie kreiert eine Angstzone.

Die Idee der »Raumgewinne« schließt an das Konzept der »national befreiten Zonen ${ }^{146}$ an. Heitmeyer unterscheidet jedoch »Raumgewinne« von »Räumungsgewinnen«, die das dauerhafte Vertreiben von Menschen aus begrenzten Sozialräumen wie Häusern beschreiben. Heitmeyer spricht bei der rassistischen Gewalt in Rostock Lichtenhagen und Hoyerswerder ${ }^{147}$ von »Räumungsgewinnen « der extremen Rechten. ${ }^{148}$ Neben »Räumungs-« und »Raumgewinnen« beschreibt Heitmeyer »Provokationsgewinne« und »Normalitäts-

139 Vgl. ebd., S. 69.

140 Auch im Nationalsozialismus wurde der Straßenraum von der NSDAP zur Repräsentation und Visualisierung von Macht verwendet. Vgl. hierzu: Schmidt, Daniel (2011): Die Straße beherrschen, die Stadt beherrschen. Sozialraumstrategien und politische Gewalt im Ruhrgebiet 1929-1933. In: Lüdtke/Reinke/Sturm (Hg.) (2011): Polizei, Gewalt und Staat im 20. Jahrhundert. Wiesbaden: VS Verlag. S. 225-248.

141 Vgl. zum Konzept der »Angstzonen«: Döring, Uta (2008): Angstzonen. Rechtsdominierte Orte aus medialer und lokaler Perspektive. Wiesbaden: Verlag für Sozialwissenschaften. S. $141 \mathrm{ff}$.

142 Vgl. Sofsky, Wolfgang (1996): Traktat über die Gewalt. Frankfurt (2005): Fischer.

143 Vgl. hierzu: Baberowski, Jörg/Metzler, Gabriele (Hg.) (2012): Gewalträume. Soziale Ordnungen im Ausnahmezustand. Frankfurt/New York: Campus.

144 Vgl. Jureit, Ulrike (2016): Umkämpfte Räume. Raumbilder, Ordnungswille und Cewaltmobilisierung. Göttingen: Wallstein. S. 13.

145 Vgl. ebd., S. 14.

146 Vgl. zu »national befreiten Zonen« außerdem Schröder, Burkhard (1997): Im Griff der rechten Szene. Reinbek: Rowohlt.

147 Vgl. zur medialen Begriffsbildung von »national befreiten Zonen«: Döring, Uta (2008): Angstzonen. Rechtsdominierte Orte aus medialer und lokaler Perspektive. Wiesbaden: Verlag für Sozialwissenschaften. S. 263.

148 Vgl. Heitmeyer, Wilhelm (1999): Sozialräumliche Machtversuche des ostdeutschen Rechtsextremismus. Zum Problem unzureichender politischer Gegenöffentlichkeit in 
gewinne«. Die von Heitmeyer beschriebenen vier Mechanismen der Eskalation sind dabei nicht linear aufeinander aufbauend zu verstehen. Sie entwickeln sich gleichzeitig und beeinflussen einander. ${ }^{149}$ Heitmeyer bezieht seine Beschreibung von »Machterweiterung durch Angstausdehnung« nicht auf Bürgerwehren, jedoch scheinen rechte Akteure, die sich als Bürgerwehr formieren, genau diese Strategie zu verfolgen. Diese Studie zeigt auf, inwiefern sich die vier Mechanismen bei den unterschiedlichen Typen von Bürgerwehren nachweisen lassen. Weil sich im Verlauf der Studie gezeigt, hat, dass die Kategorie Geschlecht auf die Motivation der Mitglieder wirkt, eine Bürgerwehr zu gründen, werden Bürgerwehren im Folgenden außerdem als vergeschlechtlichtes Phänomen in die Forschungsdebatte eingeordnet.

\subsubsection{Bürgerwehren als vergeschlechtlichtes Phänomen}

Vigilante Gruppen inszenieren sich als Beschützer und knüpfen somit an ein bestimmtes Männlichkeitsbild an - insbesondere dann, wenn sie Frauen vor anderen Männern beschützen wollen, wie bereits anhand des Beispiels des »rape myth« beschrieben. Über Männlichkeiten im deutschen Rechtsextremismus ${ }^{150}$ sowie den Zusammenhang von Geschlecht und rechtsextremer Orientierung gibt es mittlerweile wissenschaftliche Erkenntnisse. So zeigt Ursula Birsl in ihrer Studie über den Zusammenhang von der Verarbeitung geschlechtsspezifischer Sozialisationserfahrung und Rechtsextremismus auf, dass bei Männern mit rechtem Weltbild Leistungs- und Verhaltenserwartungen spezifischer Männlichkeitsnormen mit Bedürfnissen und Selbstbildern kollidieren. ${ }^{151}$

Für rechtsextreme Männer wurde lange von nur einer Form der Männlichkeit ausgegangen, von einer "gewaltbereiten Hypermaskulinität ${ }^{152}$ Ele-

Städten und Kommunen. In: Kalb, Peter/Petry, Christian/Sitte, Karin (Hg.): Rechtsextremistische Jugendliche-was tun? Weinheim: Beltz. S. 69.

149 Vgl. ebd., S. 71.

150 Vgl. bspw. Möller, Kurt (2011): Konstruktion von Männlichkeit in unterschiedlichen Phänomenbereichen des Rechtsextremismus. In: Birsl, Ursula (Hg.) (2011): Rechtsextremismus und Gender. Opladen: Budrich. S. 129-146.

151 Vgl. Birsl, Ursula (2011): Rechtsextremistisch orientierte Frauen und Männer. In: Birsl, Ursula (Hg.) (2011): Rechtsextremismus und Gender. Opladen: Budrich. S. 181.

152 Vgl. Möller, Kurt (2011): Konstruktion von Männlichkeit in unterschiedlichen Phänomenbereichen des Rechtsextremismus. In: Birsl, Ursula (Hg.) (2011): Rechtsextremismus und Cender. Opladen: Budrich. S. 129. 
mente dieser Form von Männlichkeit zeigen sich insbesondere bei männlichen Jugendlichen, die über rechtsextreme Cliquen den Einstieg in die Szene finden. ${ }^{153}$ Neuere Forschung zeigt jedoch eine ganze Bandbreite an Männlichkeitsentwürfen in rechtsextremen Milieus. ${ }^{154}$ Und auch rechtsextreme Frauen rücken mittlerweile als Täterinnen in den Fokus und mit ihnen die verschiedenen Weiblichkeiten, die in rechtsextremen Milieus performt werden. ${ }^{155}$

Für Bürgerwehren in Deutschland existieren keine Studien, die die Möglichkeit zur Performanz von Männlichkeiten als Motivation zur Teilnahme an einer Bürgerwehr untersuchen. Auch international hat die Bedeutung von Gender im Vigilantismus bisher relativ wenig Beachtung gefunden. ${ }^{156}$ Diese Studie betrachtet Bürgerwehren als Versicherheitlichungsprozess und zeigt, dass dieser stark von Männlichkeitsbildern geprägt ist.

Diese Erkenntnisse sind insbesondere für die theoretische Debatte über Theorien der Versicherheitlichung relevant (vgl. Kapitel 2.3 sowie 10.3). Die Rolle, die Geschlechterbilder in Versicherheitlichungprozessen spielen, hat bisher in der Forschungsdebatte so gut wie keine Beachtung gefunden. Eine feministische Kritik an den security studies beschreibt eine zu geringe Berücksichtigung von Frauen als Specherinnen: "Security threats specific to women, for example, tend to be marginalised because of difficulties in >being heard amongst the >speakers of security< in society. « ${ }^{157} \mathrm{Um}$ dieser Schieflage zu begegnen, wird der Ansatz der »Human Security« als eine dezidiert auch Frauen inkludierende Sicherheitstheorie eingeführt. ${ }^{158}$ Dieser

153 Vgl. Schuhmacher, Nils (2011): »Mit den Leuten zusammen kann man wirklich schon was darstellen. «. Über verschiedene Wege in rechte Jungencliquen. In: Birsl, Ursula (Hg.) (2011): Rechtsextreminsmus und Gender. Opladen: Budrich. S. 272.

154 Siehe hierzu den Sammelband von Claus/Lehnert/Müller (Hg.) (2010): »Was ein rechter Mann ist... Männlichkeit im Rechtsextremismus. Opladen: Leske + Budrich.

155 Vgl. hierzu Birsl, Ursula (2011): Rechtsextremistische Gewalt: Mädchen und junge Frauen als Täterinnen? Wissenschaftliche Erkenntnisse und offene Fragen in geschlechtervergleichender Perspektive. In: Dies. (Hg.) (2011): Rechtsextremismus und Gender. Opladen: Budrich. S. 241-264.

156 Vgl. Abrahams, Ray: Some thoughts on the comparative study of vigilantism. In: Pratten, David/Sen, Atreyee (2008): Global Vigilantes. New York: Columbia University Press. S. $437 f$.

157 Hough, Peter (2005): Who's Securing Whom? The Need for International Relations to Embrace Human Security. St Antony's International Review, 1(2). S. 83.

158 Siehe hierzu: Watson, Scott (2011): The shuman as securitization. Security Dialogue, 42(1). S. 3-20. 
Ansatz berücksichtigt jedoch nicht die Dynamiken, die Männlichkeiten in Prozessen spielen, die mit Sicherheit verbunden sind.

Es existieren zwar Studien, die Versicherheitlichungsprozesse untersuchen, bei denen ein Bereich versicherheitlicht wird, in dem Geschlecht eine relevante Kategorie darstellt - so untersucht Carpenter beispielsweise die Versicherheitlichung von "gender-based violence ${ }^{159}$ In Bezug auf die Dynamiken der Versicherheitlichung oder auf die Akteure im Versicherheitlichungsprozess wird die Kategorie Geschlecht jedoch selten analysiert. So ist es interessant, welche Rolle Geschlechterbilder für den Versicherheitlichungsprozess spielen, wenn Frauen zum Referenzobjekt ${ }^{160}$ werden und Männlichkeiten eine Rolle für die Motivation zur Versicherheitlichung und ihren Erfolg spielen. Genau diese Dynamik lässt sich bei den in dieser Studie untersuchten Bürgerwehren beobachten.

\subsubsection{Bürgerwehren als staatstheoretisch relevantes Phänomen der zivilen Sicherheitsforschung}

Zusammenfassend lässt sich festhalten, das Phänomen der Bürgerwehren wird in der Forschung in verschiedene Debatten eingeordnet. Im Rahmen der zivilen Sicherheitsforschung sind Bürgerwehren ein Ausdruck des Wandels der (Un-)Sicherheitswahrnehmungen und der zunehmenden Privatisierung der Inneren Sicherheit (Kapitel 1.2.1). Hier werden Bürgerwehren im Rahmen des Community Policing diskutiert, jedoch nur diejenigen, die mit dem Staat zusammenarbeiten beziehungsweise von ihm initiiert werden.

Es gibt jedoch bisher keine Studien im Bereich der zivilen Sicherheitsforschung, die sich mit Bürgerwehren befassen, ${ }^{161}$ die in ihrer politischen Ausrichtung und ihrem Auftreten dem Staat und seinen Werten widersprechen. Diese Bürgerwehren werden - in den wenigen Studien, die es gibt - als Phänomen des Vigilantismus staatstheoretisch analysiert (Kapitel 1.2.2).

159 Vgl. Carpenter, R. (2006): Recognizing Gender-Based Violence Against Civilian Men and Boys in Conflict Situations. Security Dialogue, 37(1). S. 83-103.

160 So zum Beispiel: Uhl, Bärbel (2014): Die Sicherheit der Menschenrechte: Bekämpfung des Menschenhandels zwischen Sicherheitspolitik und Menschenrechtsschutz (pp. 5584). Bielefeld: transcript Verlag.

161 Anika Hoffmann liefert mit ihrer kriminologischen Einordnung von Bürgerwehren jedoch einen ersten Ansatz: Hoffmann, Anika (2019): Bürgerwehren und ihre Bedeutung im öffentlichen Raum. Abweichendes Verhalten als kriminologisch relevantes Phänomen. Wiesbaden: VS. 
Das Phänomen der Bürgerwehren verdeutlicht, dass die beiden Debatten - die der zivilen Sicherheit und der staatstheoretische VigilantismusDiskurs - zusammen gedacht werden müssen. Die Forschung im Bereich der zivilen Sicherheit untersucht die (Un-)Sicherheitswahrnehmungen von Bürger*innen und die Sicherheitskultur einer Gesellschaft und befasst sich somit mit den Hintergründen für das Auftreten von Bürgerwehren. Gleichzeitig darf dabei die staatstheoretische Ebene nicht außer Acht gelassen werden. Sie ermöglicht eine Bestimmung, wann Bürgerwehren zur Sicherheit beitragen und wann sie staatliche Kontrolle infrage stellen und selbst zum Sicherheitsrisiko werden - spätestens dann, wenn sie eine rechtsextreme Strategie der Raumgewinnung darstellen (Kapitel 1.2.3).

Diese Studie leistet einen Beitrag zur zivilen Sicherheitsforschung, indem das Phänomen Bürgerwehren als Folge von Unsicherheitswahrnehmungen betrachtet wird und Bürgerwehren als Akteure des Versicherheitlichungsprozesses analysiert werden. Als rechtsextreme Strategie der Raumgewinnung stellen Bürgerwehren gleichzeitig auch eine Herausforderung für die Innere Sicherheit dar. Nicht zuletzt zeigt diese Untersuchung, dass die Bedeutung von Männlichkeiten in der Forschungsdebatte um zivile Sicherheit stärkere Beachtung finden muss. 